\title{
BTS Clinical Statement on Pulmonary Sarcoidosis
}

Thillai $\mathrm{M}^{* 1,2}$, Atkins $\mathrm{C}^{3}$, Crawshaw $\mathrm{AP}^{4}$, Hart $\mathrm{SP}^{5,6}, \mathrm{Ho} \mathrm{LP}^{7}$, Kouranos $\mathrm{V}^{8,9}$, Patterson $\mathrm{KC}^{10}$, Screaton $\mathrm{N}^{1}$, Whight $\mathrm{J}^{11}$ \& Wells $\mathrm{AU}^{* 8,9}$

* Co-chair

1. Interstitial Lung Disease Unit, Royal Papworth Hospital, Cambridge UK

2. Department of Medicine, University of Cambridge UK

3. Department of Respiratory Medicine, Norfolk and Norwich University Hospitals UK

4. Interstitial Lung Diseases Unit, University Hospitals Birmingham NHS Foundation Trust, Birmingham UK

5. Department of Respiratory Medicine, Hull University Teaching Hospitals UK

6. Respiratory Research Group, Hull York Medical School, University of Hull UK

7. Oxford Interstitial Lung Disease Service, Oxford University Hospital NHS Foundation Trust, Oxford UK

8. Interstitial Lung Disease Unit, Royal Brompton Hospital, London UK

9. National Heart and Lung Institute, Imperial College London

10. Department of Medicine, Brighton and Sussex Medical School UK

11. SarcoidosisUK

Corresponding author: muhunthan.thillai@nhs.net

\section{Contents}

1. Introduction

2. Clinical presentation

3. Cardiac sarcoidosis and pulmonary hypertension

4. Diagnosis

5. Management

6. Monitoring, discharge and withdrawal of treatment

7. Communication with patients and expert network referrals

\section{List of tables and figures}

Table 1 Staging of sarcoidosis on chest radiograph

Table 2 CT features of sarcoidosis

Table 3

CXR and CT findings to support a confident diagnosis

Table 4

Guidance for follow up

Box 1

Summary of Clinical Practice Points

Box 2 Guide to tapering and withdrawing steroid therapy.

Figure 1 Organ manifestations of sarcoidosis

Figure 2 CXR images of pulmonary sarcoidosis

Figure $3 \quad$ CT images of pulmonary sarcoidosis

Figure $4 \quad$ Suggested diagnostic approach for pulmonary sarcoidosis

Figure 5 Management of fatigue

12,504 words

152 references 


\section{Introduction}

This British Thoracic Society (BTS) Clinical Statement addresses the diagnosis, evaluation and management of pulmonary sarcoidosis, with each section summarised with key clinical practice points. In an era in which medical practice is increasingly determined by evidence-based guidelines, it must be acknowledged from the outset that current evidence in sarcoidosis, especially with regard to treatment, is weak. Thus, a number of the conclusions in this Statement are based on expert opinion and accumulated clinical experience.

The diagnosis of pulmonary sarcoidosis is often challenging, with ongoing evolution in clinician views on the need for a tissue diagnosis. Historically, a biopsy diagnosis was considered mandatory and we provide guidance on when to offer bronchoscopy and which bronchoscopic procedure to perform. We also stress that decisions made by individual patients to decline bronchoscopy, when there is a highly probable but not definite clinical diagnosis, should be supported in most cases, with careful subsequent monitoring. The document includes sections on the diagnosis of cardiac sarcoidosis and pulmonary hypertension as either disorder may present to respiratory physicians as 'symptomatic pulmonary sarcoidosis'. 
Traditional treatment algorithms and their reported application in the medical literature tend to be based on a 'one size fits all' approach and this has often led to over-treatment and major steroid-related comorbidity. However, a great many patients do not need to be treated: the broad indications for initiating therapy are a) a high risk of mortality or disability due to major organ involvement; and b) unacceptable loss of quality of life. In this statement, we focus on the management of pulmonary disease; the management of concurrent cardiac sarcoidosis or pulmonary hypertension requires referral to expert sub-specialist teams.

Key pulmonary management considerations are discussed in this statement. Whilst higher dose treatment regimens may be required in high-risk disease, a highly flexible patient-centred approach is essential when treatment is introduced solely for quality of life reasons. In this context, sustained high-dose therapy is usually inappropriate. Patients should be asked to weigh-up treatment benefits against adverse effects before longer-term treatment decisions are made, with the danger of important comorbidities arising from long-term treatment kept carefully in mind. Monitoring must be tailored to specific goals. Fatigue, a highly prevalent and often disabling symptom in sarcoidosis, requires a systematic approach. Above all, we highlight the need for active patient involvement in 
decision-making and this, in turn, requires attention to clear communication, discussed in the final part of this statement. Finally, it is important to note that in a few areas of diagnosis and management where there was a non-unanimous consensus amongst the statement authors, this is clearly indicated.

\section{Scope}

The Statement covers diagnosis and management of pulmonary sarcoidosis. Reference is also made to

diagnosis of cardiac sarcoidosis and pulmonary hypertension as either disorder may present to respiratory physicians as 'symptomatic pulmonary sarcoidosis'. Other extra pulmonary sarcoidosis diagnoses are not covered by this Statement and it is recommended that specialists with specific sarcoidosis knowledge be consulted when the disease is present outside the chest. Importantly, it may be difficult to identify the true extent of organ involvement leading to underestimation of disease outside the chest. 


\section{Methodology}

The Clinical Statement Group (CSG) was chaired by Dr Muhunthan Thillai and Professor Athol Wells. Membership was drawn from respiratory medicine, nursing, radiology and included lay/patient input. The CSG identified key areas requiring Clinical Practice Points. The overall content was developed to reflect the scope approved by the BTS Standards of Care Committee (SOCC). Following discussions of broad statement content, individual sections were drafted by group members. A final edited draft was reviewed by the BTS SOCC before posting for public consultation and peer review on the

BTS website November 2019. The revised document was re-approved by the BTS SOCC in March 2020 before final publication.

A summary of Clinical Practice points is provided in Box 1.

\section{Box 1}

Clinical Practice Points - summary

\section{Clinical Presentation}


1. The respiratory examination in pulmonary sarcoidosis is often normal, and is an unreliable measure of disease extent or morbidity.

2. Pulmonary function tests are often normal in non-fibrotic sarcoidosis, and may not reflect disease activity or symptom burden.

3. Screening for extra-thoracic disease is important. At baseline, patients should have a full blood count, biochemical tests (including urea and electrolytes, liver function tests, calcium), serum ACE levels (non-unanimous consensus) and a 12-lead ECG.

4. In patients with eye symptoms, a baseline ophthalmic review should be undertaken by either an optician or an ophthalmologist (depending on the severity of symptoms).

5. Patients should be asked routinely about fatigue and mood disturbance.

6.

comprehensive exposure and occupational history should be taken to exclude both berylliosis and silicosis which can present in a similar manner to sarcoidosis.

\section{Cardiac sarcoidosis and pulmonary hypertension}

1. Cardiac sarcoidosis and/or pulmonary hypertension should be considered in all patients with pulmonary sarcoidosis who have levels of breathlessness which are disproportionate to their lung function impairment.

2. Baseline testing in all patients with suspected cardiac sarcoidosis (i.e. those with ECG abnormalities, cardiac symptoms or breathlessness out of context with their pulmonary function) should include an ECG and echocardiogram. Abnormalities in ECG or ECHO which 
suggest cardiac sarcoidosis should be confirmed with Cardiac magnetic resonance imaging ( $\mathrm{CMR}$ ) or positron emission tomography (PET).

3. All patients with palpitations should be offered a 24 hour Holter monitor.

4. In patients with pulmonary sarcoidosis, the presence of cardiac involvement, based on advanced imaging (cardiac MRI or PET) findings, should be confirmed by a multidisciplinary team with experience in both sarcoidosis and other forms of cardiac disease. There is a current initiative to identify tertiary ILD centres that have immediate access to specialist cardiac sarcoidosis expertise.

\section{Diagnosis}

1.

patients with suspected sarcoidosis should have a CXR. If they have typical findings on a radiograph with a typical clinical presentation (eg in the context of Lofgren's disease) then a CT scan may not be necessary as long as patients are followed up in clinic with a repeat CXR within 3 months and a CT scan performed if circumstances change.

2. Multi-disciplinary review of chest imaging is recommended for all patients with a non-typical clinical presentation or CT appearance to determine the need for a confirmatory bronchoscopy or biopsy.

3. When performing a bronchoscopy, patients with predominantly lymph node disease should undergo an EBUS whereas those with predominantly parenchymal disease should have transbronchial biopsies. If both nodal and parenchymal disease is present, EBUS is the preferred initial diagnostic procedure. 
4. All patients should be part of the decision making process when deciding on whether a biopsy is necessary or whether it is safe to follow them up in the clinic alone, with a view to revisiting the need for a biopsy if circumstances change.

\section{Management}

1. There is often a fine line to making the decision to start pharmacological treatment and all patients should be fully informed and be at the heart of this decision making process.

2. Whilst there is no good evidence for any drug regimen in sarcoidosis, the majority of patients needing treatment should initially be treated with steroids ranging from $10 \mathrm{mg}$ prednisolone per day in long-standing and insidiously progressive disease, up to $20-40 \mathrm{mg}$ per day in more acute disease. A maintenance dose of 5-10 mg after these initial doses for

6-12 months is usual.

3. Most patients who need treatment should be offered methotrexate (administered orally, or subcutaneously) as the first choice of second line agent.

4. Referral for lung transplantation should be considered in all patients with advanced pulmonary fibrosis and associated pulmonary hypertension.

5. All patients with sarcoidosis related fatigue should have a systematic approach to diagnosis of the cause of fatigue and management initiated as appropriate.

6. In line with other chronic lung conditions, patients should be offered smoking cessation advice and support for anxiety or depression if needed. 


\section{Monitoring, discharge and withdrawal of treatment}

1. No patients on medication should be managed in primary care alone, even stable well patients should have hospital monitoring when on treatment. Patients under the long term care of their GP should be referred back to a hospital respiratory physician if they develop new or worsening symptoms.

2. All patients undergoing active monitoring or treatment should have regular lung function tests as part of routine care.

3. A trial of withdrawal of steroid therapy should be performed in most patients with controlled disease after 6-12 months on medication.

\section{Communication}

1. Clinicians should consider the possible need for benefits advice, occupational therapy home assessment, pulmonary rehabilitation referral as well as GP assistance with palliative care if needed e.g. in cases of progressive fibrotic pulmonary sarcoidosis with resulting respiratory failure.

2. All patients should be encouraged to complete an inventory of quality of life measures and as part of this clinicians should emphasise the concept of 'self-care' i.e. a deliberate activity that sarcoidosis patients undertake to look after their physical, mental or emotional well being 
3. There should be good communication between service providers (both between tertiary and secondary care, and between different specialists depending on organ involvement) and all patients should be offered a shared care approach where it is available. 


\section{Clinical presentation}

\section{Lung involvement in pulmonary sarcoidosis}

Sarcoidosis can affect almost any organ (Figure 1). Pulmonary involvement, including thoracic lymph node disease and/or parenchymal disease is the most commonly affected site, occurring in over $90 \%$ of cases (1-3). Bilateral hilar and right paratracheal lymphadenopathy is a classical presentation for sarcoidosis, although isolated bilateral hilar lymphadenopathy is more frequent (4). Granulomatous

inflammation most often occurs along lymphatic tracks: thus, peribronchial tissue and interlobular septa are the most common sites of sarcoid lesions. Pleural disease is uncommon (5) but should be considered in the setting of a lymphocytic effusion with other pulmonary features of the disease. Rarely, sarcoidosis affects the upper airways, including the trachea and larynx (6). Severe lower airway inflammation can result in fibrotic stenosis and distortion. (6) Pneumothoraces are a recognised complication of fibrocystic sarcoidosis (7). 
Isolated thoracic lymphadenopathy is usually asymptomatic and may be incidentally detected on imaging, as part of a screening program or after non-specific presenting symptoms such as persistent cough. In Scandinavia, a region with a high incidence of sarcoidosis, a large percentage of patients have incidental disease, with over half of all patients having Scadding stage I appearances on chest $x$-ray $(8,9)$ (see Table 1 ). In a study of incidental findings on chest CT imaging, a histological diagnosis of sarcoidosis was made in $22 \%$ of patients with at least one enlarged mediastinal lymph node ( $\geq 10 \mathrm{~mm}$ on short axis) (10).

\section{Clinical history and examination}

Most patients with node-limited disease are asymptomatic. However, some may present with systemic symptoms (fevers, night sweats, fatigue, weight loss, diffuse myalgias). Bilateral hilar lymphadenopathy (BHL) may accompany sarcoid uveitis, or parotitis. Löfgren's syndrome consists of $\mathrm{BHL}$, erythema nodosum (EN) and/or bilateral ankle arthritis. 
Patients with parenchymal sarcoidosis often present with chest symptoms, depending upon the extent of lung involvement $(1,11)$. Dyspnoea and cough are presenting features in approximately $30 \%$ of patients (2). Exertional dyspnoea tends to be mild early in disease $(1,12)$. Non-cardiac chest pain is often characterised by chest tightness or pleuritic discomfort (13). Systemic symptoms, including fatigue, are variably present when pulmonary sarcoidosis is diagnosed. Pulmonary fibrosis in sarcoidosis usually develops in long-standing, previously recognised disease, but is occasionally apparent at presentation. Cough and exertional dyspnoea are common in advanced sarcoidosis (14) and tend to be associated with sputum production when there is co-existing bronchiectasis or

aspergillus infection (15).

It is important to take an accurate exposure and occupational history to exclude conditions which may present in a similar manner including berylliosis (e.g. those in the aerospace or defence industry) and silicosis (e.g. those working in the mining, quarrying or stonemasonry industry).

The respiratory examination is almost always normal, both in node-limited sarcoidosis and in widespread nodular parenchymal disease. Wheeze and stridor are occasional features of airway 
involvement. Classical 'end-inspiratory' crackles are not a typical feature of sarcoidosis but may occur in fibrotic sarcoidosis. Clubbing is uncommon (16).

Hypoxaemia is present only in patients with extensive fibrotic disease and/or pulmonary hypertension (17). Pulmonary function tests may be normal, restrictive, obstructive or show an isolated reduction in gas transfer. Airway hyperresponsiveness is present in approximately $20 \%$ of patients, usually associated with endobronchial involvement (18). In patients with fibrotic pulmonary disease, physiologic derangements are more severe and an obstructive ventilatory defect is more

frequent (19).

Indications for diagnostic biopsy are discussed in section five. Biochemical tests are often unhelpful for diagnosis, but may serve as markers of treatment responsiveness or the need for treatment (e.g. hypercalcaemia). All patients should have a full blood count, serum calcium, liver function monitoring and an ECG. Specialist opinions should be sought if there is major extrapulmonary organ involvement. Specifically, ophthalmic review is appropriate in any patient with ocular symptoms (20) , a neurology review if any central or peripheral nervous symptoms and a dermatology review if any 
skin lesions. These opinions should be sought in parallel with management of pulmonary sarcoidosis as in some instances treatment modalities may be similar for multiple organ involvement.

All patients should have serum ACE estimation in order to identify patients in whom elevated levels are a marker of active disease and are potentially helpful during follow-up (non-unanimous consensus). 24 hour urinary Calcium estimation should not be performed in all patients but only in those with a history of renal calculi (non-unanimous consensus). In addition to basic biochemical tests, patients should have serum calcium measured at baseline. Whilst patients with sarcoidosis

may have low serum levels of 25-OH-cholecalciferol (vitamin D3), serum 1,25-(OH)2-cholecalciferol may be elevated due to granuloma macrophages overexpressing 1-alpha-hydroxylase which can lead to hypercalcaemia or hypercalciuria. If vitamin D supplementation is being considered because of deficiency or to protect bone health during oral steroid therapy, this should be carried out carefully with regular monitoring of serum and urinary calcium levels to identify and prevent significant complications of vitamin D therapy including life-threatening hypercalcaemia, renal stones and, in occasional cases, renal failure. 


\section{Fatigue and mood disturbance}

Fatigue is present in up to $80 \%$ of sarcoidosis patients, regardless of organ involvement (21), and is often difficult to manage, with a significant negative impact on patient-reported quality of life (21). The aetiology may be multifactorial. Subclinical disease activity (i.e. where sarcoidosis activity is present but below the threshold of clinical perception) (22), sleep disturbance (23), medication side-effects (24) and co-existent depression or anxiety symptoms (25) can all cause fatigue and are all

potentially modifiable. The severity and impact of fatigue varies markedly between patients with similar disease burdens.

No objective measures exist to quantify fatigue. Thus, fatigue severity is defined by patient perception, including limitation of activity. Monitoring change is equally dependent on patient reported change (significantly better/unchanged/significantly worse). Quantification of fatigue through formalised and validated questionnaire measurements (e.g. the Fatigue Assessment Scale) is needed for research but is not always helpful in managing individual patients. A number of more 
generic breathlessness scales may be used when monitoring fatigue but again none have been specifically investigated for sarcoid fatigue $(26,27)$.

\section{Clinical Practice Points}

1. The respiratory examination in pulmonary sarcoidosis is often normal, and is an unreliable measure of disease extent or morbidity

2. Pulmonary function tests are often normal in non-fibrotic sarcoidosis, and may not reflect disease activity or symptom burden

3.

Screening for extra-thoracic disease is important. At baseline, patients should have a full blood count, biochemical tests (including urea and electrolytes, liver function tests, calcium), serum ACE levels (non-unanimous consensus) and a 12-lead ECG.

4. In patients with eye symptoms, a baseline ophthalmic review should be undertaken by either an optician or an ophthalmologist (depending on the severity of symptoms)

5. Patients should be asked routinely about fatigue and mood disturbance

6. A comprehensive exposure and occupational history should be taken to exclude both berylliosis and silicosis which can present in a similar manner to sarcoidosis. 


\section{Cardiac sarcoidosis and pulmonary hypertension}

\section{Cardiac sarcoidosis}

Cardiac involvement in sarcoidosis represents a potentially dangerous form of the disease, especially when manifesting with life-threatening arrhythmias and impaired cardiac function. Cardiac

manifestations may indeed be the initial method of patients presenting with newly diagnosed sarcoidosis. This section focuses on diagnostic challenges of cardiac sarcoidosis in the context of known pulmonary involvement. The management of cardiac sarcoidosis lies beyond the scope of this statement. If diagnosed, patients should be referred to a team with specialist expertise in cardiac sarcoidosis.

The 1999 ATS/ERS sarcoidosis statement recommended screening of all patients presenting with new pulmonary sarcoidosis based on the presence of cardiac symptoms (palpitations-defined as the 
sensation of a fluttering or fast heart beat which lasts from between a few seconds to several minutes or longer, chest pain, pre-syncope/syncope) and ECG (28) but this approach has a low sensitivity (25\%) and specificity (46\%) (29) (30). This prompted the recommended addition of echocardiography (to look for cardiac dysfunction) in the presence of an abnormal ECG or a suspicion of cardiac sarcoidosis, as described by the 2014 Heart Rhythm Society (HRS) expert consensus statement (31). The use of the echocardiogram is both to look for cardiac sarcoidosis (albeit with low sensitivity) prior to a more advanced imaging modality and also to look for any other cause of cardiac dysfunction which may be seen in sarcoidosis e.g. left ventricular heart disease or

pulmonary hypertension. The HRS strategy captures clinically overt cardiac disease (conduction abnormalities, ventricular arrhythmias and new onset unexplained heart failure), present in $5-10 \%$ of the general sarcoidosis population (1), but misses sub-clinical cardiac disease, present in $20-30 \%$ of cases (29). Cardiac magnetic resonance imaging (CMR) is the diagnostic tool of choice for identification of cardiac sarcoidosis with a high diagnostic yield according to HRS criteria (sensitivity 97\% and specificity 100\%, AUC: 0.984) (30). Cardiac PET scanning also benefits from high sensitivity although the specificity is lower than CMR (29). 
Multidisciplinary discussion is currently recommended in the diagnosis of cardiac sarcoidosis (31) (32). Endomyocardial biopsy has a low yield and is seldom performed (33). Both advanced imaging modalities (CMR and PET) have considerable added value in diagnosis and prognostic evaluation but the radiation dose associated with PET should be considered if both imaging modalities are available. It is also important to note that specific patient preparation is needed for fluorodeoxyglucose (FDG)-positron emission tomography (PET) in such patients to suppress normal physiological uptake of glucose in the myocardium for image optimisation (34) (35) (36) (37). Diagnosis is based on a combination of integrating ECG/Holter (advanced atrioventricular block, ventricular tachycardia) and

echocardiographic abnormalities (left ventricular systolic or diastolic impairment) with abnormal features on advanced imaging (late gadolinium enhancement on CMR and significant FDG uptake on cardiac PET). Coronary artery disease needs to be carefully excluded as does other causes of cardiac inflammation e.g. myocarditis. Coronary artery disease should be considered in patients who have sarcoidosis and cardiac symptoms or left ventricular impairment, as patients with sarcoidosis may be at higher risk of heart disease (38). 
The selective use of CMR in patients with pulmonary sarcoidosis, based on symptomatic screening, as recommended in the ATS/ERS and HRS statements, is confounded by the presence of exertional dyspnoea due to pulmonary disease. Breathlessness can be multifactorial in pulmonary sarcoidosis. Patients with disproportionate breathlessness (i.e. not explained by pulmonary function test impairment) or loss of exercise tolerance (out of keeping with the severity of pulmonary disease and not explained by other factors such as fatigue, elevated BMI and loss of fitness) should undergo CMR, with a 24-/48- hour Holter if there are persistent palpitations. In patients with infrequent but concerning palpitations, the use of an implantable loop recorder should be considered. Given the

high prevalence of subclinical cardiac disease (29), the need for a low threshold for CMR is emphasised, if symptoms are suggestive.

However, routine CMR screening in the general sarcoidosis population has not been validated and this applies equally to PET (performed under a specific cardiac protocol), which may be used in selected patients to identify cardiac inflammation. The value of detecting limited subclinical cardiac involvement is uncertain, with no current evidence of adverse events during follow-up (39), but no definitive current data on this question. 


\section{Pulmonary hypertension [PH]}

Sarcoidosis-related pulmonary hypertension $(\mathrm{PH})$ is a significant contributor to sarcoidosis morbidity and mortality. In a recent large study, PH was confirmed by right heart catheterization in 29 of 452 patients with pulmonary sarcoidosis (6.4\%) and was a major predictor of mortality, independent of age and extent of fibrosis on HRCT (40). The five-year survival was $55 \%$ in 126 patients with PH (41) (42).

The prevalence of $\mathrm{PH}$ is critically dependent on population selection. In unselected patients attending the outpatient clinic, a prevalence of approximately $6 \%$ has been reported, (40) (43), increasing to $47 \%$ in patients with exertional dyspnoea disproportionate to pulmonary function tests, and to $74 \%$ in patients listed for lung transplantation $(44,45)$. PH tends to present with non-specific symptoms including shortness of breath, fatigue, weakness, angina and syncope.

Echocardiogram remains the most appropriate initial, non-invasive screening tool for PH. ESC/ERS guidelines recommend measurement of the tricuspid regurgitation velocity at rest, identification of 
suspected $\mathrm{PH}$ and the presence of additional echocardiographic abnormalities should be used to make a decision about the need for right heart catheterisation (46). However, echocardiographic variables are often discordant with right heart catheter measurements of pulmonary artery pressure, especially in patients with concomitant parenchymal lung disease [with one meta-analysis documenting echocardiography sensitivity of $83 \%$ and specificity of $72 \%$ for pulmonary hypertension (47)]. Therefore, referral to a PH specialist (or initial referral to a local cardiac centre with expertise in right heart catheterisation) may be warranted if indications other than echocardiographic variables (e.g. clinical features of right ventricular failure or specific ECG abnormalities) are

suggestive of PH. Unlike other forms of ILD (e.g. idiopathic pulmonary fibrosis), the use of specific treatments for $\mathrm{PH}$ may be indicated in patients with sarcoidosis.

Findings associated with $\mathrm{PH}$ include a severe reduction in DLco (Diffusing capacity for carbon monoxide) in the setting of a restrictive ventilatory defect, especially when disproportionate to lung volume reduction (46), an increase in the pulmonary artery diameter ( $\geqslant 29 \mathrm{~mm}$ ) and pulmonary artery diameter/ascending aorta diameter $(A A D)$ ratio $(\geqslant 1.0)$ (47) on CT imaging, oxygen 
desaturation $(<90 \%)$ during 6 -minute walk test $(21)$, and the need for long-term oxygen treatment (42).

$\mathrm{PH}$ more commonly represents a complication of pulmonary fibrosis than an isolated granulomatous vasculopathy (48). However, the confident identification of "disproportionate $\mathrm{PH}^{\prime}$ in the setting of chronic lung disease is notoriously difficult. Therefore, based on diagnostic, prognostic and management considerations, there should be a low threshold for referral to a PH specialist.

\section{Clinical Practice Points}

1. Cardiac sarcoidosis and/or pulmonary hypertension should be considered in all patients with pulmonary sarcoidosis who have levels of breathlessness which are disproportionate to their lung function impairment.

2. Baseline testing in all patients with suspected cardiac sarcoidosis (i.e. those with ECG abnormalities, cardiac symptoms or breathlessness out of context with their pulmonary function) should include an ECG and echocardiogram. Abnormalities in ECG or ECHO which suggest cardiac sarcoidosis should be confirmed with CMR or PET. 
3. All patients with palpitations should be offered a 24 hour Holter monitor.

4. In patients with pulmonary sarcoidosis, the presence of cardiac involvement, based on advanced imaging (cardiac MRI or PET) findings, should be confirmed by a multidisciplinary team with experience in both sarcoidosis and other forms of cardiac disease. There is a current initiative to identify tertiary ILD centres that have immediate access to specialist cardiac sarcoidosis expertise. 


\section{Diagnosis}

Imaging

Imaging is central to both the diagnosis and monitoring of pulmonary sarcoidosis patients. Typical chest radiograph findings may be highly suggestive of the diagnosis in appropriate clinical settings (Table 1) but a careful occupational history should be taken to exclude berylliosis and silicosis which can have similar CT appearances. However, thin section computed tomography (CT) is increasingly utilised to characterise subtle or non-specific findings or when there is diagnostic uncertainty. FDG

PET-CT has been proposed to assess disease activity and distribution, and to monitor therapeutic response in complex cases.

On chest radiograph, acute sarcoidosis is typically characterised by bilateral symmetrical hilar and mediastinal lymphadenopathy. Classical parenchymal changes include mid and upper zone predominant nodular or reticulonodular opacity and upper zone predominant fibrosis. However, the chest radiograph is normal in $20 \%$ of cases. Radiographic staging of sarcoidosis has prognostic value 
(Figure 2) but is limited by inter-observer agreement. Surveillance chest radiography in evolving cases every 3-6 months is typically used for patient monitoring given its low radiation dose.

Table 1 Staging of sarcoidosis on a chest radiograph

\begin{tabular}{|c|c|c|c|}
\hline Scadding stage & 4 & 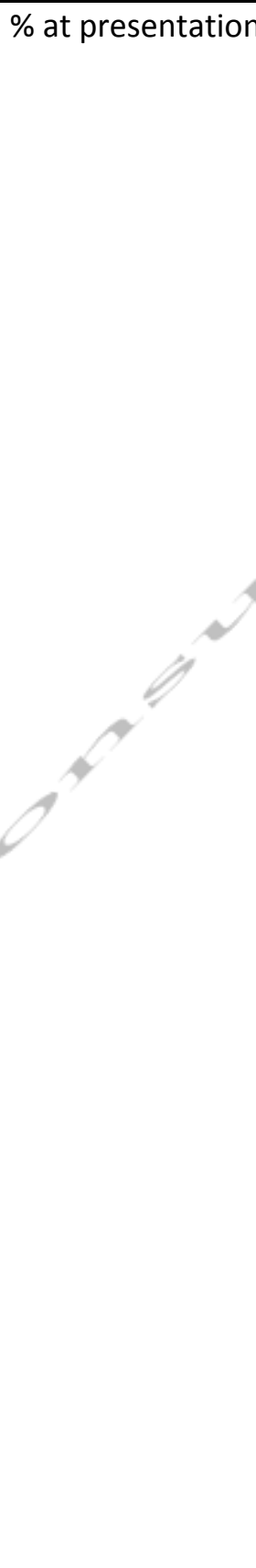 & $\begin{array}{l}\% \text { with clinical and } \\
\text { radiographic } \\
\text { resolution untreated } \\
\end{array}$ \\
\hline 0 & Normal & $5-15$ & $\mathrm{n} / \mathrm{a}$ \\
\hline 1 & Enlarged nodes only & $45-65$ & $50-90$ \\
\hline II & $\begin{array}{l}\text { Enlarged nodes and } \\
\text { parenchymal changes }\end{array}$ & $30-40$ & $30-70$ \\
\hline III & $\begin{array}{l}\text { Parenchymal changes without } \\
\text { enlarged nodes or fibrosis }\end{array}$ & $10-15$ & $10-20$ \\
\hline
\end{tabular}




\begin{tabular}{|l|l|l|l|}
\hline IV & Fibrosis & 5 & 0 \\
\hline
\end{tabular}

CT is more sensitive than chest radiography in characterising lymphadenopathy, particularly nodal calcification, and in detecting and characterising lung parenchymal features. Thus, CT is especially helpful in diagnosis when there is clinico-radiological discordance or when radiographic features are atypical. The presence of typical CT features (Figure 3) may prevent the need for invasive sampling (Table 2). However, a range of atypical CT features of sarcoidosis have led to its description as "the great pretender" (50). CT is more discriminatory than chest radiography in identifying pulmonary

fibrosis, and may provide prognostic information (51) and help to guide therapeutic escalation or rationalisation depending on clinical scenarios. CT scans can also guide both endobronchial ultrasound-guided nodal sampling and transbronchial biopsy where required. Most sarcoidosis patients with clinically overt interstitial lung involvement will need a CT scan. However, it can be argued that in patients with typical clinical and chest radiographic features, CT is not required provided that there is no evidence of disease progression during follow-up (Table 3 ). 
Surveillance CT should be limited to patients with unexplained deterioration in symptoms or lung function, those with "red flags" such as haemoptysis or pulmonary hypertension or when specific conditions are suspected radiographically such as aspergilloma. 
Table 2 CT scanning features of sarcoidosis

\begin{tabular}{|c|c|}
\hline \multicolumn{2}{|l|}{ Typical CT features } \\
\hline \multicolumn{2}{|l|}{ ypical ci realuies } \\
\hline Lymph node enlargement & $\begin{array}{l}\text { Hilar +/- mediastinal } \\
\text { Bilateral, symmetrical } \\
\text { Well defined homogeneous }\end{array}$ \\
\hline Lymph node calcification & 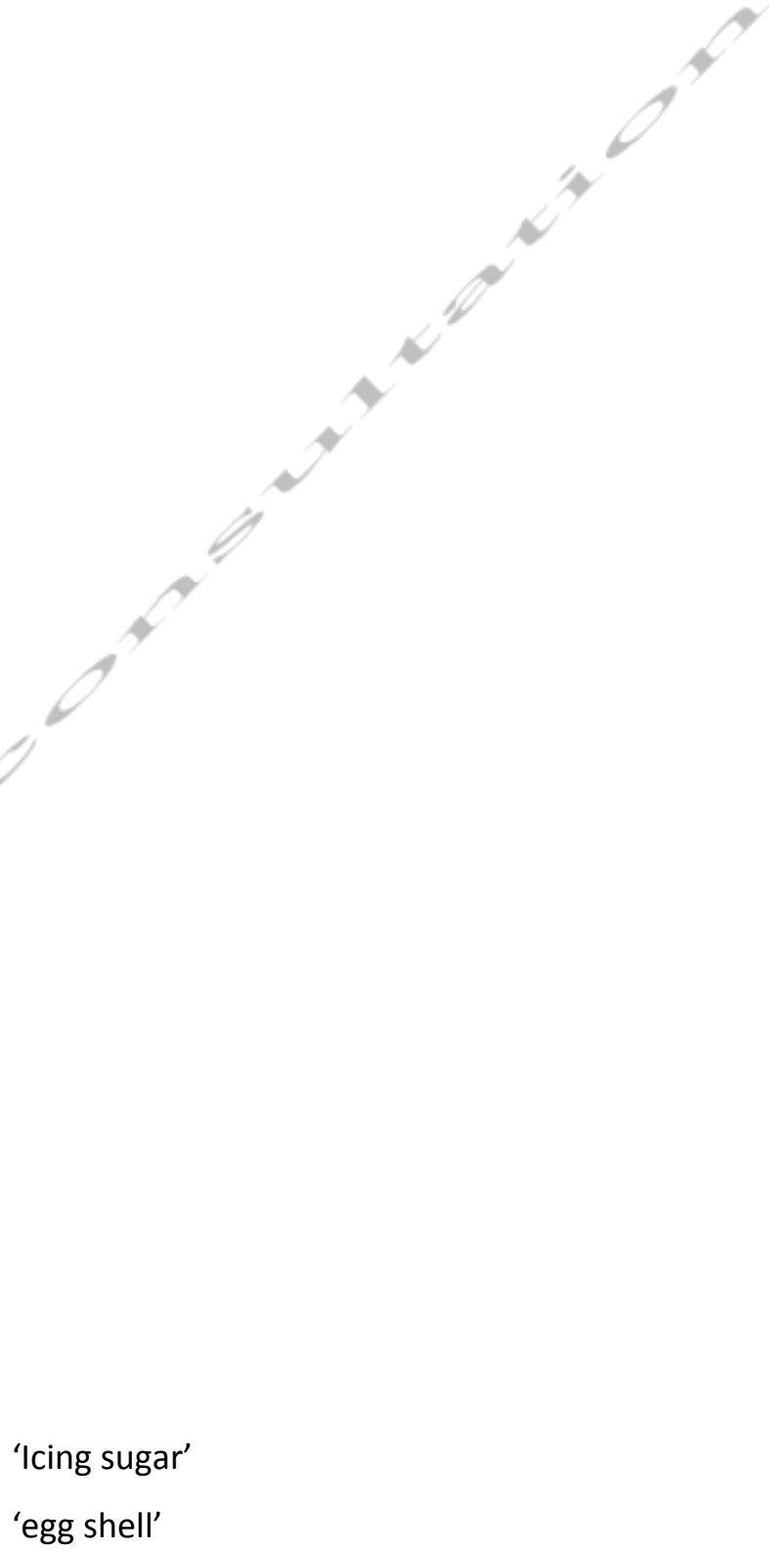 \\
\hline Nodules & $\begin{array}{l}\text { Well defined 2-5mm } \\
\text { Paraseptal predominance } \\
\text { Fissural beading / peribronchovascular nodularity } \\
\text { Coalescence - larger nodules / perihilar } \\
\text { consolidation }\end{array}$ \\
\hline Fibrosis & Reticular opacity \\
\hline
\end{tabular}




\begin{tabular}{|c|c|}
\hline & $\begin{array}{l}\text { Volume loss / Architectural distortion } \\
\text { Traction bronchiectasis } \\
\text { Peribronchovascular }\end{array}$ \\
\hline \multirow{2}{*}{\multicolumn{2}{|c|}{ Air trapping / Mosaic pattern }} \\
\hline & \\
\hline Chest site & Feature \\
\hline Lymph node enlargement & Unilateral / asymmetric \\
\hline $\begin{array}{l}\text { Confluent consolidation / Ground glass } \\
\text { opacity }\end{array}$ & ( \\
\hline \multicolumn{2}{|l|}{ Solitary / multiple discrete nodules } \\
\hline \multicolumn{2}{|l|}{ Miliary nodularity } \\
\hline Bullae / cysts / aspergilloma & Upper zone predominant \\
\hline Pleural thickening / effusion / plaques & \\
\hline
\end{tabular}


Table 3 CXR and CT findings to support a confident diagnosis

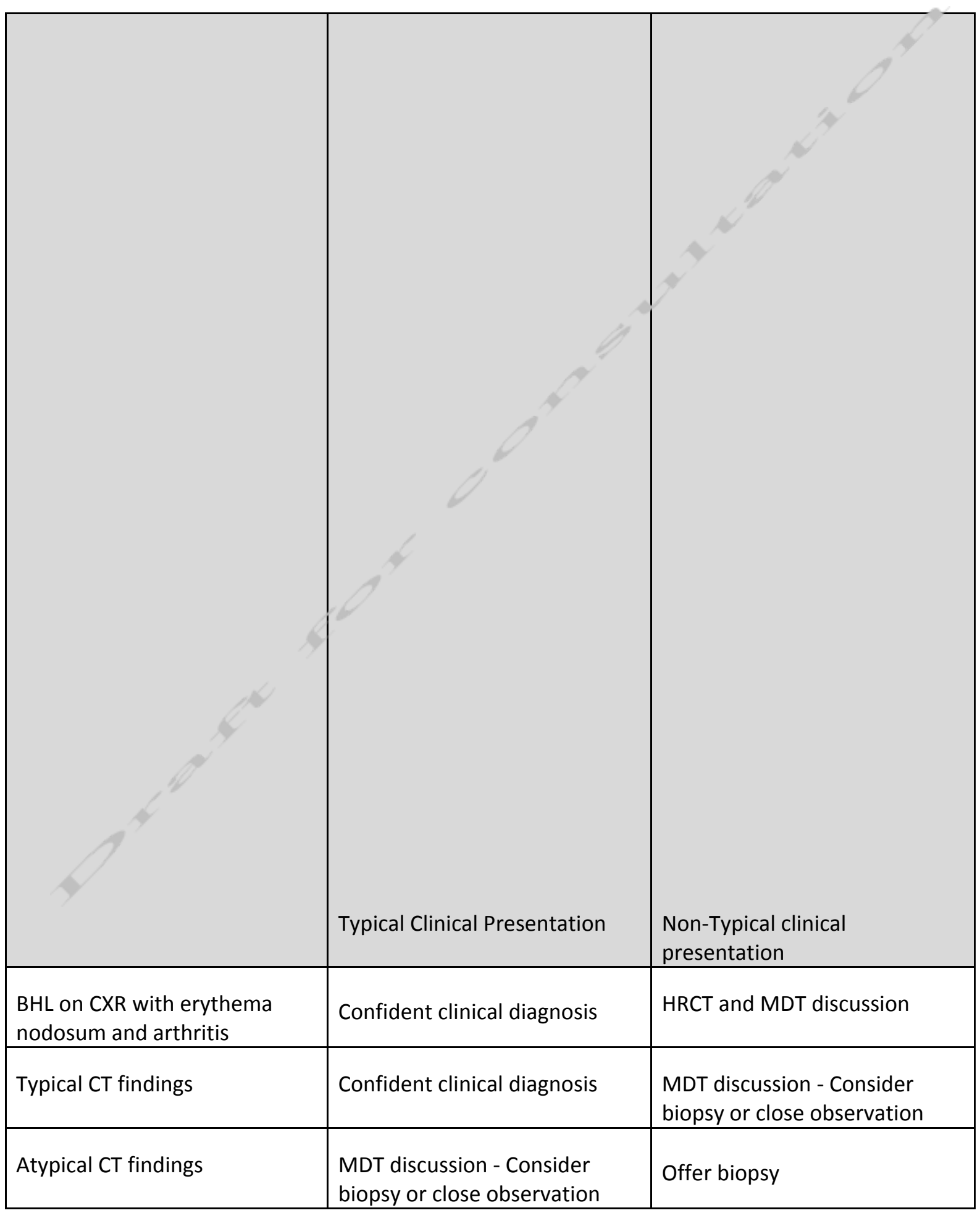


CXR= Chest radiograph $(x-$ ray $)$

$\mathrm{HRCT}=$ High resolution $\mathrm{CT}$ (computerised tomography) scan

MDT $=$ Multidisciplinary meeting

FDG PET is a sensitive means of detecting sarcoidosis inflammation (52). The Royal College of Radiologists 2016 PET-CT guidelines indicate that PET CT can be used in the assessment of sarcoidosis disease activity in highly selected cases when there is diagnostic uncertainty using conventional imaging, particularly where cardiac sarcoidosis is suspected (53).

\section{Bronchoscopy and biopsy}

An absolute diagnosis of sarcoidosis requires a tissue biopsy. However, there are two scenarios in which one is not needed to make a confident diagnosis (Figure 4).

- In patients with Lofgren's syndrome and no overt alternative diagnosis, close monitoring is appropriate with biopsy required only if atypical features emerge during follow-up (54). 
- In patients with long-standing pulmonary disease following a typical clinical presentation and stable typical imaging findings, an alternative diagnosis is highly unlikely. In such cases an MDT discussion of the clinical case may be needed in order to make the diagnosis with confidence.

These two scenarios aside, the diagnosis of sarcoidosis should not routinely be made on clinical grounds alone, particularly if pharmacological treatment is needed or if there is diagnostic uncertainty, i.e. a need to exclude a) lymphoma, tuberculosis or carcinoma where there are enlarged

lymph nodes; or b) other forms of interstitial lung disease (ILD). Tissue should be obtained from the most accessible site, for example a skin lesion or peripheral lymph node if abnormal findings are present. If no peripheral site is present then intrathoracic sampling is indicated via fibreoptic bronchoscopy. It is essential that the patient be involved in this decision-making process. Bronchoscopy carries a specific morbidity risk and this should be clearly explained to patients. Some patients may opt for careful follow up without an immediate biopsy, with biopsy undertaken at a later date if the disease behaviour is not in keeping with sarcoidosis. 
Endobronchial appearance at bronchoscopy is normal in up to two thirds of patients with sarcoidosis (55). Nodules, classically 2-3mm waxy yellow mucosal lesions with a mucosal cobblestone appearance, should be biopsied at bronchoscopy $(56,57)$. Bronchoalveolar lavage (BAL) is a useful diagnostic adjunct: an elevated BAL lymphocyte count of $15-25 \%$ provides support for granulomatous disease (including sarcoidosis but also seen in accelerated silicosis) whereas a count $>50 \%$ is more suggestive of hypersensitivity pneumonitis or cellular non-specific interstitial pneumonia. However an accurate cell differential count may not be possible in all hospital pathology departments (58). A CD4:CD8 ratio $>4$ in the absence of an increased proportion of other

inflammatory cell types is highly specific (>95\%) for sarcoidosis when compared with other inflammatory ILDs $(12,59,60)$. The BAL should be performed in a 'high volume' manner i.e. installation of 100 to $300 \mathrm{mls}$ into a distal airspace as per ATS guidance for ILD bronchoscopy (12) and then samples sent for a cell differential count (local expertise permitting) and microscopy and culture (including an examination for acid fast bacilli) to rule out infection as an alternative diagnosis or complication. There is no validated role for repeated BAL in disease monitoring (61).

The yield from endobronchial biopsy (EBB) is $40-60 \%$, even in airways which macroscopically appear 
normal although this yield may be lower (55) and in transbronchial biopsy (TBBx) has been reported as $40-90 \%$ with a pneumothorax rate of $1-9 \%$ in diffuse ILD (62). Whilst the current BTS advice remains to perform TBBx under fluoroscopy guidance (63), there is no evidence that this either reduces the rate of pneumothorax or increases diagnostic yield in diffuse lung diseases (63-67).

Endobronchial ultrasound (EBUS)-guided transbronchial node aspiration (EBUS-TBNA) with the addition of EUS-TBNA (endoscopic ultrasound-TBNA) has revolutionised the diagnosis of nodal disease over and above the use of conventional TBNA (68-71). The diagnostic yield in sarcoidosis in

meta-analyses ranged from $54 \%$ to $93 \%$ with a pooled sensitivity of $79 \%$. The varied yields are likely to be explained by variability in the staging, number of samples taken and the variable use of ROSE (rapid onsite 'pathologic' examination) $(72,73)$. Until recently, the utility of EBUS-TBNA in sarcoidosis continued to be debated $(74,75)$. However, the GRANULOMA trial showed that in stage I/II disease, the EBUS-TBNA yield was $74 \%$ compared to $48 \%$ with EBB/TBBx $(p<0.01)$ (76). A subsequent randomised controlled trial revealed that EBUS-TBNA had the highest diagnostic yield (74.5\%), which was improved over CTBNA $(48.4 \%, \mathrm{P}=0.004)$ or EBB $(36.3 \%, \mathrm{P}<0.0001)$. The addition of TBBx (but not EBB) significantly enhanced the yield of EBUS-TBNA but this needs to be balanced 
against the increased time and risk needed for the procedure (77). Based on current evidence, an EBUS-TBNA is an appropriate primary diagnostic approach for stage I and II sarcoidosis, variably combined with EBB, TBBx and BAL (78) as detailed in figure 4.

Cryobiopsy, a recently developed technique which can be performed on self-ventilating patients under deep sedation, allows the retrieval of significantly larger samples without the crush artefact seen in TBBx (79-81). Safety concerns include pneumothorax and the potential for significant bleeding, especially in less experienced hands. The added value of cryobiopsy over EBUS-TBNA +-

TBBx in the diagnosis of sarcoidosis is unclear, particularly when weighing up the balance of yield and procedural risk.

If conventional bronchoscopic biopsies are non-diagnostic, the clinician (or MDT) must decide how far to pursue a tissue diagnosis. For example, a patient with a non-diagnostic EBUS-TBNA and diagnostic uncertainty may be invited to undergo mediastinoscopy, or a TBBx (if sufficient parenchymal disease). By contrast, in a patient with probable sarcoidosis and a non-diagnostic 
EBUS-TBNA (with sufficient tissue to 'rule out' lymphoma and carcinoma) a clinical diagnosis of sarcoidosis might be made. It is essential that the wishes of the fully informed patient be taken into account in this decision-making process.

\section{Clinical Practice Points}

1. All patients with suspected sarcoidosis should have a CXR. If they have typical findings on a radiograph with a typical clinical presentation (eg in the context of Lofgren's disease) then a CT scan may not be necessary as long as patients are followed up in clinic with a repeat CXR within 3 months

and a CT scan performed if circumstances change.

2. Multi-disciplinary review of chest imaging is recommended for all patients with a non-typical clinical presentation or CT appearance to determine the need for a confirmatory bronchoscopy or biopsy.

3. When performing a bronchoscopy, patients with predominantly lymph node disease should undergo an EBUS whereas those with predominantly parenchymal disease should have transbronchial biopsies. If both nodal and parenchymal disease is present, EBUS is the preferred initial diagnostic procedure. 
4. All patients should be part of the decision making process when deciding on whether a biopsy is necessary or whether it is safe to follow them up in the clinic alone, with a view to revisiting the need for a biopsy if circumstances change.

\section{Management}

\section{Overview of outcomes and broad indications for treatment}

Sarcoidosis is widely viewed as a benign disease, based on good outcomes in the majority of patients, with regression or stabilisation of pulmonary disease. However, in recent cohorts of sarcoidosis patients, sarcoidosis itself has been the single most frequent cause of death, with mortality clearly exceeding that in matched general populations (82) (42) (83). In one cohort, the need to introduce sarcoidosis-specific therapy within three months of diagnosis was a marker for a major increase in mortality ( $\mathrm{HR} 2.34$ [95\% Cl 1.99-2.75]) (82). Overall, in 6-8\% of patients with sarcoidosis, there is a reduction in life expectancy due to the disease. Pulmonary involvement (ILD 
and/or pulmonary hypertension) causes up to $70 \%$ of sarcoidosis-attributable fatalities with cardiac involvement accounting for most remaining deaths (84).

However, these data may seriously understate mortality linked to sarcoidosis as fatal comorbidities due to sarcoidosis treatment are not considered. Comorbidities found with sarcoidosis include coronary artery disease, stroke or transient ischaemic attacks, arthritis, depression, diabetes, hypertension and major osteoporotic fractures, all of which are associated with increased hospitalization (38) (85) (86) (87) (88). Their effect on quality of life and mortality is well

documented, with linkages between fatal outcomes and both the number of comorbidities and a comorbidity burden score in sarcoidosis (87) (88). Steroid usage in sarcoidosis was a strong determinant of a high composite comorbidity score (that included diabetes, hypertension, weight gain, hyperlipidaemia and osteoporosis) (86). It is also likely that a proportion of infective deaths in treated patients are directly due to steroid or immunosuppressive treatment.

Significant pulmonary, hepatic and renal disease are readily identified by recommended screening tests (28), although cardiac involvement is sometimes difficult to detect. Neurosarcoidosis is usually 
clinically overt. It has been estimated that approximately $30 \%$ of patients with sarcoidosis have clinically significant major organ involvement (89). Long term mortality due to sarcoidosis approximates $20-25 \%$ (i.e. $6-8 \% / 30 \%$ ) when there is overt major organ involvement. This proportion increases when treatment-related mortality is taken into account (84).

Given the need to minimise treatment-related comorbidities in sarcoidosis, we recommend that treatment should be initiated only if there is a) potential danger of a fatal outcome or permanent disability; or b) unacceptable loss of quality of life. The approach to treatment differs according to the

indication. Historically, most recommended regimens have consisted of initial high dose steroid therapy, followed by a prolonged consolidative approach (e.g. Prednisolone 10-20mg daily). However, higher dose regimens, needed in patients at risk of death or permanent disability, are often counterproductive when treatment is introduced for quality of life reasons. Unfortunately, side-effects are sometimes more debilitating than the symptoms for which treatment has been introduced, with a net reduction in quality of life. 
The accurate introduction of therapy requires careful risk stratification, with the identification of patients with advanced disease (dangerous sarcoidosis) and also patients at higher risk of progression to advanced disease (potentially dangerous sarcoidosis). Advanced pulmonary disease is associated with a major increase in mortality despite treatment (51) (40) (90). Therefore, treatment should be introduced earlier in the course of disease to pre-empt this situation. Indications for treatment in pulmonary disease, based on disease severity, are discussed in detail below.

\section{The treatment of sarcoidosis-related morbidity}

With the exclusion of dangerous/potentially dangerous disease, the introduction of treatment for loss of quality of life is critically dependent on the values and choices of the patient and must take into consideration their wishes. The patient is the only person with a true perception of the impact of sarcoidosis symptoms on their daily life. Loss of quality of life results from a "package" of symptoms, which varies widely between patients. Importantly, pulmonary sarcoidosis is often associated with a combination of respiratory and systemic symptoms. Disabling fatigue is perhaps 
the single most frequent source of unacceptable loss of quality of life (91) and is addressed in detail later in this statement.

It is important that whilst initial investigations to screen for dangerous/potentially dangerous disease are undertaken, the patient is asked to consider carefully whether the impact of sarcoidosis on daily life is sufficiently debilitating to cause them to choose to be treated. It must be stressed that accurate management is not dependent solely on medical expertise but requires the empowerment of patients in decision-making. In essence, this is a risk-benefit discussion, with the

decision made by the patient informed by expert knowledge of potential benefits and treatment side-effects.

Historical high-dose treatment regimens geared to dangerous/potentially dangerous sarcoidosis are unsuited to treatment aimed at improving morbidity. The choice and dose of agent should be negotiated with the patient with the understanding that the patient should have the freedom to cautiously modify the starting regimen, within its therapeutic range, by titration against symptoms and side-effects. Patient empowerment allows a wide array of arrangements to be made with 
different patients, with the possible introduction of low dose steroids at variable doses, hydroxychloroquine or, even, a second-line agent such as methotrexate, either alone or in combination. The patient, as the sole judge of the impact of morbidity, should be given the opportunity to take the lead in treatment decisions, in contrast to the treatment of dangerous/potentially dangerous disease. However, it must also be expected that some patients will opt to be wholly guided by medical advice.

The desire to empower the patient must be balanced against the obligation of the treating physician to

consider potential medication toxicities, and therefore a truly informed discussion should be undertaken. This allows on the one hand patient concerns about steroid therapy to be addressed, with critical differences between low and high dose steroid regimen explained, and if necessary, the use of alternative agents from the outset. On the other, it provides meaningful context for a "slow hand" approach, in cases in which the clinical presentation may not justify the risk of potentially toxic therapies.

\section{Pharmacological treatment}


The first treatment decision is whether or not to introduce immunosuppressive drugs (ie those which suppress the immune system). This is influenced by the presence of significant pulmonary dysfunction, unacceptable loss of quality of life and the views of the patient. This statement focuses on the treatment of aggressive or persistent pulmonary disease. In reality, many patients with major pulmonary disease also have other organ involvement and/or debilitating symptoms and require careful nuancing of therapy on a case by case basis.

Disease-modifying drugs can be divided into three sections: a) steroids (e.g. Prednisolone), b) classical immunosuppressants (most commonly Methotrexate, Azathioprine, Leflunomide and Mycophenolate) and c) biologics (e.g. Infliximab). Usual international practice, based on biological rationale and accumulated clinical experience, has been to initiate treatment with Prednisolone. It should be acknowledged that this has never been definitively validated by controlled data. Despite a multiplicity of reports, steroid therapy has never been evaluated according to specific treatment indication (severe pulmonary disease as opposed to impaired quality of life). Many studies include consecutive patients with active sarcoidosis, including those with mild disease severity and little or 
no symptom burden. With these caveats, the current statement accords with the existing expert consensus. It is stressed that this decision should be multidisciplinary with the integration of all the tabulated variables.

\section{Steroid regimens}

The use of exact pulmonary function test thresholds is made difficult by individual variations and baselines. For example, the use of a pulmonary function test threshold (e.g. Dlco $<60 \%$ of predicted) is made more difficult by the normal pre-morbid range of $80-120 \%$ of predicted - thus, a value of

$60 \%$ in an individual patient may indicate a real reduction to anywhere between $25 \%$ and $50 \%$ of the pre-morbid value. Given this constraint, treatment should be considered when there is significant reduction in pulmonary function tests e.g. Dlco $<65 \%$, spirometric volumes $<70 \%$ or if there is a $10 \%$ drop from baseline FVC or $15 \%$ drop from baseline Dlco. The caveat is presence of inactive fibrotic disease which may have resulted in irreversible but non-progressive lung function loss. In some cases, patients with progressive breathlessness due to pulmonary disease (with the careful exclusion of other causes of breathlessness e.g. cardiac disease) may be suitable for steroid initiation despite not meeting a significant reduction in pulmonary function tests but this should be evaluated on an 
individual basis with clear discussion between the clinician and the patient. Good control of disease is shown by regression of disease or lack of progression, as judged by symptoms, radiologic imaging and pulmonary function variables.

The exact dose of prednisolone depends upon the clinical setting. When disease is long-standing and insidiously progressive, lower dose protective therapy from the outset may be appropriate (e.g. Prednisolone 10mg daily). Aggressive therapy with intravenous methylprednisolone is seldom warranted in pulmonary disease in isolation but may be required with rapidly progressive

extra-pulmonary disease (e.g. cardiac sarcoidosis, neurosarcoidosis, severe optic neuritis).

In many cases, the decision to treat is a fine line and needs to be negotiated with the patient where patient views often determine the immediate approach. If treatment is withheld, it is helpful to reach agreement that treatment will be introduced if there is evidence of further disease progression. 
In the absence of life-threatening disease, initial treatment comprises $20-40 \mathrm{mg} /$ day Prednisolone for 4-6 weeks, followed by slow tapering (e.g. reducing by $5 \mathrm{mg}$ every 2 weeks) to a maintenance dose, usually between $5-10 \mathrm{mg} / \mathrm{d}$. The aim is to bring disease under control and to maintain this control until the threat to the organ is resolved.

Subsequent treatment is critically dependent upon the initial response. If there is major regression of disease, or residual disease is only moderately severe, a rapid reduction to low dose steroid therapy can be justified. However, a lengthy maintenance period is often required. Some patients

have persistent, severe chronic disease: under-treatment in this context risks further irreversible disease progression, yet a preventative effect of treatment has not been definitely established. The BTS study of Gibson and colleagues suggests that approximately $40 \%$ of patients will spontaneously remit within the first 6 months, but it is not clear which individual patients will do this and which will have progressive chronic disease (92). Adequate bone protection should be considered in all patients on long term steroids. 
In the longer term, a target dose of 5-10 mg/day is broadly appropriate but ongoing side-effects such as weight gain and osteoporosis may require individualisation of the dose. Continued treatment is indicated if withdrawal or dose reduction is associated with relapse (with a brief return to higher dose treatment and tapering to the previous dose if the relapse is major). Attempts at withdrawing Prednisolone should be made every 6-12 months. The potential development of major comorbidities due to steroid therapy should be kept constantly in mind.

Löfgren syndrome occurs in up to $30 \%$ of patients and remits in $70 \%-80 \%$ within two years (93).

Although organs are seldom threatened (some patients can be managed with NSAIDs alone for arthralgias), systemic symptoms (especially severe arthralgia) often require steroid therapy. However, treatment is usually needed for less than three months, with higher doses (e.g. $30-40 \mathrm{mg} /$ daily) for $1-2$ weeks and tapering within weeks to months. Paradoxically there is a small sub-group of patients who may do worse with steroid treatments and these patients should be identified with steroids withdrawn early if this is suspected (93). 
Analysis of the British Thoracic Society Sarcoidosis Registry showed that $8 \%$ of all patients with pulmonary sarcoidosis were prescribed inhaled steroids (94). Although an obstructive defect is present in almost a quarter of UK patients with sarcoidosis (95), there is no evidence for inhaler efficacy (96). Thus, this statement does not recommend their routine use in a diagnosis of sarcoidosis alone.

\section{Second-line immunosuppression}

Before second line agents are introduced, the diagnosis of sarcoidosis should be reviewed and compliance with therapy should be discussed in a sensitive manner. The need for second line agents will usually indicate referral to a specialist tertiary centre but clinicians in secondary care with a strong experience of their use will be well placed to initiate treatment and monitor these patients.

Indication for the addition of second-line agents include: 
a. progression of pulmonary disease or an unacceptable symptom burden despite adequate steroid therapy

b. intolerable steroid side effects

c. inability to taper steroid below 10 to $15 \mathrm{mg} /$ day (97) (98) (99) (100)

d. The presence of major comorbidities likely to be adversely affected by steroid therapy (severe obesity, diabetes mellitus, osteoporosis, hypertension).

e. A strong patient aversion to the use of steroids, in which case, a second line agent may occasionally be used as initial therapy.

Absence of consensus on the definition of disease phenotypes, treatment indications and validated outcome measures contributes to the paucity of evidence to support second line treatment recommendations (101) Options for escalation include methotrexate (used most frequently), mycophenolate, leflunomide and azathioprine. All of these agents carry a not insubstantial risk of toxicity including, but not limited to myelosuppression, hepatotoxicity, opportunistic infection and 
implications for those planning conception. For these reasons, all immunosuppressive drugs need blood test monitoring and whilst this is usually defined in local centres, one acceptable regimen will be for full blood count, renal and liver blood monitoring every 2 weeks on initiation of drug and then (if levels are normal and stable) a reduction in frequency after 2 months to monthly testing and then a further reduction after 6 months to blood testing every 3 months (102).

The side effect rate of these drugs are variable: methotrexate (18\%), mycophenolate $(21 \%)$, leflunomide (34\%) and azathioprine (35\%) (103) (104) (105). Prior to treatment with any of the

following agents a full blood profile, renal function, liver function and viral hepatitis serology should be measured. Those with an eGFR of less than 30 or with an aminotransferase level above two times the upper limit of normal (unless this is solely due to sarcoidosis itself), or chronic infection with hepatitis B or C should not be candidates for these treatments.

Methotrexate, is an antimetabolite drug and the most widely used agent second line agent for pulmonary sarcoidosis enabling a reduction or cessation of steroids, although it must be acknowledged that there is no data clearly showing superiority to azathioprine (i.e. either agent can 
be justified as first choice second-line immunosuppression, with individual side-effect profiles on initial use often highly influential). In retrospective studies (which should be cautiously interpreted), methotrexate has had an efficacy of $50 \%$ and response rate of $40-60 \%$, using FVC and DLCO as outcome measures, (106) (107) (108) (109) (110) (103).

Methotrexate is administered orally, or subcutaneously in cases of refractory nausea or insufficient response at six months, is typically initiated at $5-10 \mathrm{mg} /$ week and incrementing fortnightly to a target of $15-20 \mathrm{mg}$ per week as tolerated (110). Folic acid should be prescribed routinely ( $5 \mathrm{mg}$ weekly) to

reduce the incidence of myelosuppression.

Methotrexate-induced pneumonitis is rare in sarcoidosis but when it occurs it may be difficult to distinguish from progressive interstitial lung changes. A recent large randomised controlled trial of methotrexate in cardiac disease found that the incidence of acute pneumonitis was $0.2 \%$ (111). It is characterised by a non-productive cough, dyspnoea, and fever, which can occur acutely i.e. within days to weeks after initiation, or can occur insidiously. The development of new ground-glass on chest radiography or CT is suggestive, especially when changes differ in morphology from 
pre-existing imaging abnormalities. Poorly formed granulomas may be seen on lung biopsy although fibrosis is not a feature. Recovery usually occurs after withdrawal of the drug.

Other significant complications include hepatic fibrosis (in up to 10 percent of cases when the cumulative dose exceeds $5 \mathrm{~g}$, roughly equivalent to two years therapy at standard doses), leukopenia, nausea, alopecia and skin rash (108).

Azathioprine affects RNA and DNA synthesis thereby inhibiting lymphocyte proliferation, a key feature of sarcoidosis immune pathology although the exact mechanism of action in sarcoidosis is not

clear (109). There are no randomised trials of azathioprine in pulmonary sarcoidosis but open-label series and cohort study suggest a modest improvement (103). The usual starting dose of azathioprine is $50 \mathrm{mg}$ per day, increased by $25 \mathrm{mg}$ every two to three weeks until the maintenance dose is reached, typically $2 \mathrm{mg} / \mathrm{kg}$. The most common side effects include nausea, vomiting, diarrhoea, rash, fever and malaise. Pancytopenia, which can be difficult to distinguish from lymphopenia associated with sarcoidosis, abnormal liver function and an increased risk of subsequent malignancy have been reported. TPMT serum levels should be measured to ensure they are not low prior to starting azathioprine. No significant differences in efficacy were seen between 
methotrexate and azathioprine in a direct comparison except for a higher rate of infection with azathioprine (35 versus 18 percent), possibly reflecting the selective use of prophylactic antibiotics with methotrexate treatment (103).

Mycophenolate mofetil (MMF), an inhibitor of lymphocyte proliferation and activity, is used to treat a variety of connective tissue disease-associated ILDs associated and is generally well tolerated (112). Data regarding the use of MMF in sarcoidosis are limited and it should not considered before methotrexate or azathioprine unless there is a specific reason. However, one retrospective analysis in

pulmonary sarcoidosis suggested that MMF may be of benefit in those who have failed an initial second line treatment and may, in addition, enable steroid reduction (105). Doses of 1-1.5g twice daily are typically used. Neutropenia is less of a problem with MMF than with other immunosuppressive agents, but may occur. Nausea and diarrhoea may be dose limiting and it has potential teratogenicity in women of child bearing age, as do other second line agents.(113).

Leflunomide, an antimetabolite similar to methotrexate but with less gastrointestinal toxicity, may be used alone or with methotrexate. Experience is largely extrapolated from its use in rheumatoid 
arthritis, but small case series data suggest a beneficial effect on FVC and steroid reduction with leflunomide (104) (105) (114) (115). The most common adverse effects of leflunomide are nausea, diarrhoea, abdominal pain, hypertension, hepatotoxicity, rash, and peripheral neuropathy.

Cyclophosphamide is an alkylating agent that is metabolised by the cytochrome P-450 system into active metabolites which decrease lymphocyte numbers and function and may also have anti-inflammatory effects. Despite being overall one of the most commonly used immunosuppressive agents, it is rarely used as a steroid-sparing agent in the treatment of

sarcoidosis due to its toxicity profile.

Hydroxychloroquine is advocated primarily for use in fatigue, joint and skin sarcoidosis but it may be used as an adjunct when helping patients wean off higher doses of prednisolone. The usual dose is $200 \mathrm{mg}$ once or twice a day. Retinal and cardiac toxicities are rare but potentially serious. An ophthalmic examination is recommended at the time of treatment initiation (or within 12 months of starting treatment) in all patients in whom more than five years treatment is likely to be needed. 
Patients should then be referred for annual review after five years of treatment (116). Patients should also have a baseline ECG to exclude long QT interval.

All patients on long term immunosuppression should be counselled against the risk of repeated infections. If this occurs, patients may need prophylactic antibiotics (including against PJP). All forms of immunosuppression should also be reviewed for their potential for drug interactions with existing medications and additionally dose reductions may need to be used if patients have liver or renal disease.

\section{Biological agents and antifibrotics}

Biological agents are considered third line therapeutic agents, to be initiated in pulmonary disease only after a failure of second line treatment. In the UK, these are not routinely available and can only be prescribed through specialist tertiary centres.

Tumour necrosis factor (TNF) is a pro-inflammatory cytokine thought to accelerate the inflammatory process in sarcoidosis via its role in maintenance of granuloma formation. Thus, using agents that block the effect of TNF may be beneficial in treating sarcoidosis, particularly in a subset of patients 
with $\mathrm{CD4}^{+}$Iymphopenia (117). Infliximab given in combination with methotrexate or azathioprine appears to improve disease control (118) (119) (117). The major adverse effects include increased susceptibility to infection, particularly mycobacterial and invasive fungal infections, infusion-reactions, alopecia, oral candidiasis, visual field defect and increased rate of fatal pulmonary embolism. Paradoxically, the development of non-caseating granulomata consistent with sarcoidosis has been reported during anti-TNF therapy for other diseases (120). Patients should be screened for latent TB infection (including the use of Interferon Gamma Release Assay testing) prior to starting anti-TNF agents (121). If latent TB is detected, patients should have anti-TB treatment as per local

guidelines for treatment of latent TB infection prior to starting biologic therapy. Infliximab is given initially every 2 weeks and then every $4-8$ weeks as part of maintenance therapy.

The antifibrotic medications pirfenidone and nintedanib are currently only available in the United Kingdom for Idiopathic Pulmonary Fibrosis (IPF) $(122,123)$. However, the recent INBUILD study reviewed patients with non-IPF fibrotic lung disease including NSIP (Nonspecific interstitial pneumoinia), hypersensitivity pneumonitis and sarcoidosis. Although the numbers of sarcoidosis patients in the study was small, there was an overall reduction in the annual rate of decline in FVC in 
patients treated with nintedanib vs controls, paving the way for the use of antifibrotics in progressive fibrotic lung diseases including sarcoidosis, once other immunosuppressive therapies have been exhausted (124).

\section{Lung transplantation}

In those with advanced pulmonary fibrosis and/or associated pulmonary hypertension, lung

transplantation may be considered. The indications for lung transplantation in sarcoidosis are outside the scope of this statement but one approach would be to follow that similar to indications for Idiopathic Pulmonary Fibrosis i.e. a significant acute (e.g. 10\% over 6 months) drop in FVC or DLCO in association with respiratory failure (125). Bilateral lung transplantation appears to be associated with slightly better survival than single lung transplantation (126) (45) (127). Following lung transplantation, asymptomatic foci of non-caseating granulomas suggestive of recurrent disease have been identified in the allografts of sarcoidosis patients, but clinically significant organ dysfunction due to recurrent sarcoidosis is rare (128) (126). 


\section{Management of fatigue}

When managing a patient with sarcoidosis-associated fatigue, alternative causes should be excluded (figure 5). Anaemia, vitamin D deficiency, iron deficiency, thyroid dysfunction or hypercalcaemia may all contribute to symptoms but as described earlier careful consideration should be given to vitamin D supplementation and monitoring to prevent toxicity. Screening for sleep disorders, including obstructive sleep apnoea, insomnia and periodic limb movements, should be undertaken if symptoms suggestive of these conditions are present. Sleep disorders may develop due to

steroid-induced weight gain. Depression and anxiety may co-exist and exacerbate fatigue severity. Patients with sarcoidosis and fatigue may also display skeletal muscle weakness (129). Multiple aetiologies may therefore co-exist in an individual patient.

If no reversible non-sarcoidosis cause can be identified, strategies for directly managing or reducing fatigue should be initiated if there is unacceptable loss of quality of life. A short trial of steroid therapy may be helpful (non-unanimous consensus) in order to identify the minority of patients in whom fatigue is controllable by anti-inflammatory treatment, based on a) patient assessment of 
impact on their daily lives; and b) the judgment by the treating physician that this intervention is not outweighed by potential harm, taking into account comorbidities such as osteoporosis, high BMI, diabetes and hypertension.

There was no group consensus on whether a treatment trial should consist only of low dose steroid therapy (e.g. Prednisolone 5-10mg daily) or an initial challenge with higher dose treatment. When fatigue is steroid responsive, low dose maintenance therapy (e.g. prednisolone 5-10mg daily) may be appropriate with the exact dose determined by titration by patients against symptoms.

Hydroxychloroquine in combination with steroids may be beneficial but this is anecdotal at best (130).

Ginkgo Biloba has been promoted as a natural remedy for fatigue. It is widely available and is well tolerated aside from an increased risk with concurrent anticoagulation (131-133), although evidence for efficacy is poor and it has not been trialled in patients with sarcoidosis. In refractory fatigue neurostimulants can be used for symptomatic relief but these are currently used off-licence. Methylphenidate and modafinil have been studied in small numbers of patients with sarcoidosis; 
both appear to be beneficial for fatigue and were well tolerated over a short period but are used off licence and should be used in caution in patients with psychiatric diseases including depression. $(134,135)$.

Non-pharmacological management strategies and therapies may complement pharmacological interventions. Fatigue diaries and pacing techniques enable patients to identify the severity and variability of their fatigue, and to structure activity within their physical and mental capabilities. Structured exercise routines, similar to pulmonary rehabilitation, have shown evidence of benefit in

some patient groups and patients with progressive pulmonary sarcoidosis may be suitable for standardised pulmonary rehabilitation programmes (136), but this may not be suitable for all. Advice on exercise therapy from physiotherapists as part of the multidisciplinary team may be helpful in some cases. Mindfulness techniques have also been shown in one small study to be helpful in sarcoid fatigue (137).

It is likely that a combination of the suggested options may be required, with an individual strategy devised for each patient. It is important to discuss the various treatment options with the patient 
and make a joint decision on the management plan, included goals of therapy, with regular planned review and monitoring of fatigue.

\section{Clinical Practice Points}

1. There is often a fine line to making the decision to start pharmacological treatment and all patients should be fully informed and be at the heart of this decision making process.

2.

Whilst there is no good evidence for any drug regimen in sarcoidosis, the majority of patients needing treatment should initially be treated with steroids ranging from $10 \mathrm{mg}$ prednisolone per day in long-standing and insidiously progressive disease, up to $20-40 \mathrm{mg}$ per day in more acute disease. A maintenance dose of 5-10 mg after these initial doses for 6-12 months is usual.

3. Most patients who need treatment should be offered methotrexate (administered orally, or subcutaneously) as the first choice of second line agent.

4. Referral for lung transplantation should be considered in all patients with advanced pulmonary fibrosis and associated pulmonary hypertension. 
5. All patients with sarcoidosis related fatigue should have a systematic approach to diagnosis of the cause of fatigue and management initiated as appropriate.

6. In line with other chronic lung conditions, patients should be offered smoking cessation advice and support for anxiety or depression if needed.

6. Monitoring, discharge and withdrawal of treatment

\section{Role of monitoring}

Patients with sarcoidosis should be monitored to a) observe spontaneous resolution in recently diagnosed cases, b) look for progression or confirm improvement or stability in untreated patients, c) determine the effect of anti-inflammatory treatment and d) look for relapse in patients who are tapering or have discontinued therapy. A realistic outcome target should be decided jointly by the clinician and patient at the outset, and may include resolution, improvement, or stability, with or without anti-inflammatory therapy. 


\section{Symptoms}

Symptoms and their impact on quality of life should be assessed at every visit, with reference to pre-defined individualised goals. In steroid-treated patients, changes in symptoms are the prime guide to escalation or withdrawal of treatment. Assessment may be complicated by adverse effects of steroid therapy which overlap with features of sarcoidosis, such as fatigue and sleep disturbance. Similarly, intercurrent illness such as respiratory infection can contribute to symptoms. Therefore, if disease progression is suspected corroboration is required, initially with chest X-ray and pulmonary

function testing. Consideration should be given to cardiac sarcoidosis, pulmonary embolism (138) or pulmonary hypertension if breathlessness is worsening.

\section{Lung function tests}

Lung function tests are used to monitor changes in airflow obstruction, pulmonary restriction, or low gas transfer. There are no thresholds that define a significant change in lung function in sarcoidosis, but in fibrotic lung diseases a fall of $10 \%$ from baseline in FVC or $15 \%$ for DLco indicate that change is 
not due to measurement variability. In sarcoidosis these thresholds should not be used in isolation but should be integrated with symptomatic and with possible imaging changes.

Disproportionate serial reduction in FEV1 may denote increasing airflow obstruction due to bronchial involvement $(139,140)$ despite stable chest X-ray appearances. The FVC is sensitive to changes in disease severity in patients with ILD, and is usually concordant with changes in DLco, and may function as a similar marker of disease progression in sarcoidosis specifically (141). A falling DLco associated with stable spirometry should raise the suspicion of pulmonary hypertension.

\section{Chest radiographs}

Increasing lung opacities seen on chest X-ray are seen in many (but not all) patients with worsening pulmonary sarcoidosis, and chest X-ray deterioration correlates with worsening symptoms and declining lung function (142). However, a change in Scadding X-ray stage is uncommon and is an insensitive marker of change (143). In patients withdrawing from steroid therapy, chest X-ray deterioration can be used to detect relapse before symptoms worsen (144). 


\section{Computed tomography}

Serial CT imaging should not be performed routinely but repeat CT can be valuable for assessing suspected disease progression when there is discordance between symptoms, chest X-ray, and physiology. Review of chest imaging in a multi-disciplinary format is recommended. The focus should be on assessing changes in the lung parenchyma which indicate disease progression (145). Lymph node enlargement in isolation is not a marker of progression. CT scoring systems (146) are of research interest but remain to be validated in practice.

\section{Serum ACE and other disease activity markers}

Measures of granulomatous inflammatory 'activity' in sarcoidosis including serum ACE activity, BAL lymphocytes, and ${ }^{67}$ Gallium scanning correlate poorly with disease progression and have little value in monitoring sarcoidosis (non-unanimous consensus) or predicting treatment response (147). Even in apparently self-resolving disease, serum ACE activity may remain elevated but this is not a cause for concern. Markers of disease activity are often suppressed with anti-inflammatory therapy and do not predict risk of relapse. 


\section{Other blood tests}

Because of the risk of hypercalcaemia, serum calcium should be monitored, particularly in summer months when production of cholecalciferol is highest due to skin exposure to UVB light. Serum liver function tests are useful for monitoring patients with hepatic sarcoidosis, but it is unusual for clinically significant liver disease to arise de novo in patients with normal liver function tests early in the disease course.

\section{Extra-pulmonary sarcoidosis}

When significant extra-pulmonary disease is present involvement of the relevant specialists and multidisciplinary discussions for decision making are recommended.

\section{Withdrawal of treatment}


A trial of withdrawal of steroid therapy is typically performed after 6-12 months in patients with controlled disease. Monitoring of treatment tapering and withdrawal focusses on detecting worsening disease, which must be distinguished from adrenal insufficiency or steroid withdrawal syndrome.

Risk of relapse is higher in patients who have received steroid therapy than in treatment-naïve patients, with reported relapse rates of $13-75 \%$ when anti-inflammatory treatment is withdrawn

(142). Importantly, most relapses occur within 1 year of discontinuing therapy. Relapsing disease probably represents sarcoidosis that never truly resolved, but which was temporarily suppressed by steroid therapy.

\section{Steroid withdrawal symptoms}

Steroid withdrawal syndrome describes patients who suffer symptoms on steroid withdrawal in the presence of normal hypothalamic-pituitary-adrenal (HPA) function. Slow tapering may not prevent withdrawal symptoms which include fatigue, dizziness, headache, mood swings, arthralgia, myalgia, 
nausea, and abdominal pain. In contrast to adrenal insufficiency, steroid withdrawal symptoms may occur even when dose reduction occurs in the supra-physiological range (>7.5mg prednisolone daily) but at these doses patients are not at risk of adrenal crisis. In sarcoidosis it can be challenging to distinguish increasing fatigue or myalgia due to steroid withdrawal from worsening disease, with the risk that the steroid dose is increased and patents continue on long term steroids unnecessarily $(92$, 148). Steroid withdrawal symptoms are self-limiting and success depends on perseverance with the lowest possible steroid dose and then proceeding with a very slow taper over several months (149) see Box 2.

\section{Adrenal insufficiency}

Adrenal insufficiency should always be taken seriously (150). There is considerable individual variation in adrenal recovery following steroid treatment, and the dose and duration of therapy are poor predictors of subsequent adrenal insufficiency. Generally, long term prednisolone doses $\leq 7.5$ mg daily are less likely to cause adrenal suppression (151). Biochemical insufficiency detected with a cosyntropin (synacthen) suppression test does not necessarily translate into clinical meaningful adrenal failure but may become clinically relevant during periods of stress and illness. 


\section{Box 2. Guide to tapering and withdrawing steroid therapy.}

- Gradually taper steroid dose over months to allow recovery of HPA axis

- Reduce steroid dose to physiologic range (5-7.5mg prednisolone daily)

- Reduce prednisolone from $5 \mathrm{mg}$ od by $1 \mathrm{mg}$ every 2 weeks to $1 \mathrm{mg}$; then $1 \mathrm{mg}$ alternate days for 2 weeks

- Stop treatment

- Warn patient that steroid supplementation with short term steroids may be needed to cover illness or surgery for up to 1 year following cessation of therapy

For a patient with steroid-withdrawal symptoms or suspicion of adrenal insufficiency

- Consider alternate day therapy or convert to daily hydrocortisone

- Measure 8-9am cortisol (note cross reactivity of synthetic steroids with cortisol assay). Can be performed pre-dose ( $24 \mathrm{~h}$ post dose) prednisolone

- Morning cortisol $<100 \mathrm{nmol} / \mathrm{L}$ suggests adrenal insufficiency; $>450 \mathrm{nmol}$ per litre is consistent with adrenal sufficiency. If 100-450 $\mathrm{nmol} / \mathrm{L}$ consider stimulation test and specialist endocrinology referral

- If cortisol insufficient, maintain on physiological dose with advice to increase dose 2-3x to cover injury or illness. After 3 months, perform further adrenal stimulation testing and if cortisol sufficient, slowly taper dose.

Adapted from Hopkins (151) and Iliopoulou (149). 


\section{Duration of follow-up and discharge}

Patients with Lofgren's syndrome or stage I chest X-ray can be discharged from medical care once resolution is confirmed. More frequent and longer term monitoring is recommended for patients with more severe disease and a higher risk of progression (Table 4). Patients in whom steroid therapy has been withdrawn are at highest risk of worsening and require the most frequent monitoring, particularly during the year after cessation. Patients should not be discharged to primary care if there is dangerous or potentially dangerous disease, major ongoing loss of quality of life or the

patient is still on pharmacological treatment for sarcoidosis.

Table 4. Guidance for follow-up. Adapted from Valeyre (152)

\begin{tabular}{|l|l|l|l|}
\hline & Frequency & Duration & Discharge \\
\hline $\begin{array}{l}\text { Lofgren's syndrome or } \\
\text { stage I chest X-ray }\end{array}$ & 6 monthly & 2 years & Yes \\
\hline Stage II-IV chest X-ray & $\begin{array}{l}3-6 \text { monthly or } \\
\text { Annually (depending } \\
\text { on clinical suspicion }\end{array}$ & $\begin{array}{l}2 \text { years or } \\
\text { Long term }\end{array}$ & No \\
\hline
\end{tabular}




\begin{tabular}{|l|l|l|l|}
\hline & $\begin{array}{l}\text { of a change in } \\
\text { disease behaviour) }\end{array}$ & Long term & No \\
\hline $\begin{array}{l}\text { Significant } \\
\text { extra-pulmonary } \\
\text { disease }\end{array}$ & $3-6$ monthly & $\begin{array}{l}\text { 1 year or } \\
\text { Minimum 3 years after } \\
\text { cessation }\end{array}$ & No \\
\hline $\begin{array}{l}\text { Withdrawal from } \\
\text { steroid therapy }\end{array}$ & $\begin{array}{l}2-3 \text { monthly or } \\
3-6 \text { monthly }\end{array}$ & \\
\hline
\end{tabular}

\section{Clinical Practice Points}

2. No patients on medication should be managed in primary care alone, even stable well patients should have hospital monitoring when on treatment. Patients under the long term care of their GP should be referred back to a hospital respiratory physician if they develop new or worsening symptoms.

2. All patients undergoing active monitoring or treatment should have regular lung function tests as part of routine care. 
3. A trial of withdrawal of steroid therapy should be performed in most patients with controlled disease after 6-12 months on medication. 


\section{Communication with patients and expert network referrals}

\section{Discussion with patients}

Patients with sarcoidosis are eager for information about the cause, prognosis, expected long-term course, and treatment options. Online, self-directed searches for information yield an array of results, sometimes conflicting, which often provokes anxiety and confusion. Clinicians should clarify and help untangle this web of information. However, it can be challenging to convey the disease process when the cause remains unknown, and the use of disease phenotype to inform

prognostication, whilst important, is limited by incomplete data. In addition, the try-and-see treatment approach for immunosuppressive therapies, which results in variable and often unpredictable outcomes, can be frustrating.

Explaining the disease process of sarcoidosis is not easy. The unknown antigen factor may cause reflection on possible environmental or occupational exposures. Yet, there is a dearth of data regarding the timing and nature of the sarcoid antigen exposure. In addition, the largely unmeasurable contribution of genetic, epigenetic, and other factors to disease susceptibility is an 
unsatisfying disease model. Acknowledging this frustration and leaving space for patient questions reflects compassion on behalf of the care provider. Clinicians should also actively signpost further sources of information which are directly targeted at patients including those from the British Lung Foundation and SarcoidosisUK.

Outcomes in sarcoidosis vary by organ phenotype. The extent and anatomical topography of disease, as well as response to treatment, are key prognostic features. Therefore, optimal counselling acknowledges data from large scale epidemiology reports but contextualizes results to individual

factors. For example, emphasising that most patients in pooled cohorts enter remission within a few years of diagnosis can be misleading for patients with severe disease, such as aggressive cutaneous disease, neurosarcoidosis, or extensive pulmonary involvement; for the latter, the onset of fibrosis will especially influence long-term outcomes. In contrast, reported outcomes for cardiac sarcoidosis are alarming, yet not all patients experience significant disability or premature death. Many subjects in earlier reports were not on treatment or had not undergone screening for an internal cardiac defibrillator. For pro-actively managed patients, an individual's clinical status is a critical determinant of long-term outcomes. 
As sarcoidosis is a dynamic and incurable disease, treatment decision making is a long-term process rather than a one-time task. Explaining the triggers for treatment helps patients understand why not everyone requires treatment, or why treatment may be indicated long after diagnosis. As reviewed in this document, the decision to treat or not is based on the status of disease balanced against possible side effects of therapies. When treatment is warranted, patient counselling should include the rationale for a long-term course, including the plan for staged dose adjustments based on treatment effect and medication tolerance. The profile of side effects can be overwhelming. A printed summary or handout, in simple and easy to read language, for patients to refer to after the

visit is invaluable. Side effect monitoring is often labour intensive for the patient and clinical practice alike; establishing a mechanism for patients to readily access office staff to report concerns or provide testing updates is essential.

When the diagnosis of sarcoidosis is certain (without concern for another environmental or occupational disease, such as berylliosis or hypersensitivity pneumonitis) there is no indication for patients to change their daily activities for the purpose of improving sarcoidosis. However, medical care for sarcoidosis also can be a valuable window of opportunity to motivate patients to overcome 
a sedentary lifestyle, pursue a healthy diet and regular exercise, and cease smoking or recreational drug use. These interventions help with sarcoidosis-associated fatigue and body aches, mitigate the weight-gain risk of systemic steroid treatment, and avoid further lung injury. Referral to a General Practitioner for further advice on healthy lifestyle may be helpful for many patients.

\section{Interactions between secondary and tertiary care}

All patients with sarcoidosis, regardless of presenting organ phenotype, should undergo surveillance for

multi-organ disease for at least a few years following the initial diagnosis. Preparing patients for this course underscores the chronic disease model of sarcoidosis. Patients with active sarcoidosis are typically followed by an expert consultant in a tertiary ILD unit, who can confidently assess the diagnosis, and establish treatment and long-term monitoring plans with the support of an expert multidisciplinary team. At this point it is crucial that patients have access to specialist respiratory nurses who can assist with drug monitoring, managing side effects and help with access to other care providers. Such access could be either in person or through the use of nurse-led telephone clinics. 
In multi-organ disease, several providers may be involved. Consultants perform organ-specific tests to confirm sarcoidosis and to rule out disease mimics. When treatment extends beyond systemic immunosuppression, consultants also manage organ specific medications, such as topical treatments for cutaneous disease. Sarcoidosis management is complex, particularly for active and multi-system disease, and good communication among medical providers is essential. A lead clinician should be identified to both the patient and the medical team as the person to supervise care. A robust approach to communication keeps other involved providers informed of important treatment decisions and recent test results. In some instances, a consultant appointment may be overwhelming for

patients, and it is not reasonable to assume that they can relay accurate information to other providers. It is therefore vital that there is good communication between primary, secondary and tertiary care and patients should be offered a shared care approach (i.e. care between centres) if they wish and that the infrastructure is available for this.

\section{Clinical Practice Points}


1. Clinicians should consider the possible need for benefits advice, occupational therapy home assessment, pulmonary rehabilitation referral as well as GP assistance with palliative care if needed e.g. in cases of progressive fibrotic pulmonary sarcoidosis with resulting respiratory failure.

2. All patients should be encouraged to complete an inventory of quality of life measures and as part of this clinicians should emphasise the concept of 'self-care' i.e. a deliberate activity that sarcoidosis patients undertake to look after their physical, mental or emotional well being

3. There should be good communication between service providers (both between tertiary and secondary care, and between different specialists depending on organ involvement) and all patients should be offered a shared care approach where it is available.

\section{Acknowledgements}

To be completed.

\section{Disclaimer}

A Clinical Statement reflects the expert views of a group of specialists who are well versed on the topic concerned, and who carefully examine the available evidence in relation to their own clinical practice. A Clinical Statement does not involve a formal evidence review and is not developed in accordance with clinical practice guideline methodology. Clinical Statements are not intended as 
legal documents or a primary source of detailed technical information. Readers are encouraged to consider the information presented and reach their own conclusions.

\section{Declarations of interest}

Declarations of interest were completed in line with the BTS Policy and are available from the BTS Office on request. 


\section{References}

1. Baughman RP, Teirstein AS, Judson MA, Rossman MD, Yeager H, Jr., Bresnitz EA, et al. Clinical characteristics of patients in a case control study of sarcoidosis. Am J Respir Crit Care Med. 2001;164(10 Pt 1):1885-9.

2. Mana J, Rubio-Rivas M, Villalba N, Marcoval J, Iriarte A, Molina-Molina M, et al. Multidisciplinary approach and long-term follow-up in a series of 640 consecutive patients with sarcoidosis: Cohort study of a 40-year clinical experience at a tertiary referral center in Barcelona, Spain. Medicine (Baltimore). 2017;96(29):e7595.

3. Hattori T, Konno S, Shijubo N, Yamaguchi T, Sugiyama Y, Honma S, et al. Nationwide survey on the organ-specific prevalence and its interaction with sarcoidosis in Japan. Sci

Rep. 2018;8(1):9440.

4. Greco FG, Spagnolo P, Muri M, Paladini I, Chizzolini F, Piciucchi S, et al. The value of chest radiograph and computed tomography in pulmonary sarcoidosis. Sarcoidosis Vasc Diffuse Lung Dis. 2014;31(2):108-16.

5. Szwarcberg JB, Glajchen N, Teirstein AS. Pleural involvement in chronic sarcoidosis detected by thoracic CT scanning. Sarcoidosis Vasc Diffuse Lung Dis. 2005;22(1):58-62.

6. Polychronopoulos VS, Prakash UBS. Airway involvement in sarcoidosis. Chest. 2009;136(5):1371-80.

7. Manika K, Kioumis I, Zarogoulidis K, Kougioumtzi I, Dryllis G, Pitsiou G, et al. Pneumothorax in sarcoidosis. J Thorac Dis. 2014;6(Suppl 4):S466-9. 
8. Hillerdal G, Nou E, Osterman K, Schmekel B. Sarcoidosis: epidemiology and prognosis. A 15-year European study. Am Rev Respir Dis. 1984;130(1):29-32.

9. Pietinalho A, Ohmichi M, Hirasawa M, Hiraga $Y$, Lofroos AB, Selroos O. Familial sarcoidosis in Finland and Hokkaido, Japan--a comparative study. Respir Med. 1999;93(6):408-12.

10. Stigt JA, Boers JE, Oostdijk AH, van den Berg JW, Groen HJ. Mediastinal incidentalomas. J Thorac Oncol. 2011;6(8):1345-9.

11. Loddenkemper R, Kloppenborg A, Schoenfeld N, Grosser H, Costabel U. Clinical findings in 715 patients with newly detected pulmonary sarcoidosis--results of a cooperative study in former West Germany and Switzerland. WATL Study Group. Wissenschaftliche

Arbeitsgemeinschaft fur die Therapie von Lungenkrankheitan. Sarcoidosis Vasc Diffuse Lung Dis. 1998;15(2):178-82.

12. Meyer KC, Raghu G, Baughman RP, Brown KK, Costabel U, du Bois RM, et al. An official American Thoracic Society clinical practice guideline: the clinical utility of bronchoalveolar lavage cellular analysis in interstitial lung disease. Am J Respir Crit Care Med. 2012;185(9):1004-14.

13. Hendrick DJ, Blackwood RA, Black JM. Chest pain in the presentation of sarcoidosis. Br J Dis Chest. 1976;70(3):206-10. 
14. Nardi A, Brillet PY, Letoumelin P, Girard F, Brauner M, Uzunhan Y, et al. Stage IV sarcoidosis: comparison of survival with the general population and causes of death. Eur Respir J. 2011;38(6):1368-73.

15. Bonham CA, Strek ME, Patterson KC. From granuloma to fibrosis: sarcoidosis associated pulmonary fibrosis. Curr Opin Pulm Med. 2016;22(5):484-91.

16. Kumar R, Goel N, Gaur SN. Sarcoidosis in north Indian population: a retrospective study. Indian J Chest Dis Allied Sci. 2012;54(2):99-104.

17. Baughman RP, Shlobin OA, Wells AU, Alhamad EH, Culver DA, Barney J, et al. Clinical features of sarcoidosis associated pulmonary hypertension: Results of a multi-national registry. Respir Med. 2018;139:72-8.

18.

Shorr AF, Torrington KG, Hnatiuk OW. Endobronchial involvement and airway hyperreactivity in patients with sarcoidosis. Chest. 2001;120(3):881-6.

19. Yeager H, Rossman MD, Baughman RP, Teirstein AS, Judson MA, Rabin DL, et al. Pulmonary and psychosocial findings at enrollment in the ACCESS study. Sarcoidosis Vasc Diffuse Lung Dis. 2005;22(2):147-53.

20. Salah S, Abad S, Brezin AP, Monnet D. [Sarcoidosis]. J Fr Ophtalmol. 2019;42(3):303-21.

21. Drent M, Lower EE, De Vries J. Sarcoidosis-associated fatigue. Eur Respir J. 2012;40(1):255-63. 
22. Teirstein AS, Machac J, Almeida O, Lu P, Padilla ML, lannuzzi MC. Results of 188 whole-body fluorodeoxyglucose positron emission tomography scans in 137 patients with sarcoidosis. Chest. 2007;132(6):1949-53.

23. Bosse-Henck A, Wirtz H, Hinz A. Subjective sleep quality in sarcoidosis. Sleep Med. 2015;16(5):570-6.

24. Fleischer M, Hinz A, Brahler E, Wirtz H, Bosse-Henck A. Factors associated with fatigue in sarcoidosis. Respir Care. 2014;59(7):1086-94.

25. de Kleijn WP, Drent M, De Vries J. Nature of fatigue moderates depressive symptoms and anxiety in sarcoidosis. Br J Health Psychol. 2013;18(2):439-52.

26. Tanizawa K, Handa T, Nagai S, Oga T, Kubo T, Ito Y, et al. Can the Sarcoidosis Health

Questionnaire predict the long-term outcomes in Japanese sarcoidosis patients? Respir Med. 2019;149:1-8.

27. Patel AS, Siegert RJ, Creamer D, Larkin G, Maher TM, Renzoni EA, et al. The development and validation of the King's Sarcoidosis Questionnaire for the assessment of health status. Thorax. 2013;68(1):57-65.

28. Statement on sarcoidosis. Joint Statement of the American Thoracic Society (ATS), the European Respiratory Society (ERS) and the World Association of Sarcoidosis and Other Granulomatous Disorders (WASOG) adopted by the ATS Board of Directors and by the ERS Executive Committee, February 1999. Am J Respir Crit Care Med. 1999;160(2):736-55. 
29. Mehta D, Lubitz SA, Frankel Z, Wisnivesky JP, Einstein AJ, Goldman M, et al. Cardiac involvement in patients with sarcoidosis: diagnostic and prognostic value of outpatient testing. Chest. 2008;133(6):1426-35.

30. Kouranos V, Tzelepis GE, Rapti A, Mavrogeni S, Aggeli K, Douskou M, et al. Complementary Role of CMR to Conventional Screening in the Diagnosis and Prognosis of Cardiac Sarcoidosis. JACC Cardiovasc Imaging. 2017;10(12):1437-47.

31. Birnie DH, Sauer WH, Bogun F, Cooper JM, Culver DA, Duvernoy CS, et al. HRS expert consensus statement on the diagnosis and management of arrhythmias associated with cardiac sarcoidosis. Heart Rhythm. 2014;11(7):1305-23.

32. Kouranos V, Sharma R, Wells AU. Accurate diagnosis of cardiac sarcoidosis needs a

multidisciplinary approach. Br J Hosp Med (Lond). 2016;77(11):614-5.

33. Uemura A, Morimoto S, Hiramitsu S, Kato Y, Ito T, Hishida H. Histologic diagnostic rate of cardiac sarcoidosis: evaluation of endomyocardial biopsies. Am Heart J. 1999;138(2 Pt 1):299-302.

34. Patel MR, Cawley PJ, Heitner JF, Klem I, Parker MA, Jaroudi WA, et al. Detection of myocardial damage in patients with sarcoidosis. Circulation. 2009;120(20):1969-77.

35. Youssef G, Leung E, Mylonas I, Nery P, Williams K, Wisenberg G, et al. The use of 18F-FDG PET in the diagnosis of cardiac sarcoidosis: a systematic review and metaanalysis including the Ontario experience. J Nucl Med. 2012;53(2):241-8. 
36. Blankstein R, Osborne M, Naya M, Waller A, Kim CK, Murthy VL, et al. Cardiac positron emission tomography enhances prognostic assessments of patients with suspected cardiac sarcoidosis. J Am Coll Cardiol. 2014;63(4):329-36.

37. Kandolin R, Lehtonen J, Airaksinen J, Vihinen T, Miettinen H, Ylitalo K, et al. Cardiac sarcoidosis: epidemiology, characteristics, and outcome over 25 years in a nationwide study. Circulation. 2015;131(7):624-32.

38. Bargagli E, Rosi E, Pistolesi M, Lavorini F, Voltolini L, Rottoli P. Increased Risk of Atherosclerosis in Patients with Sarcoidosis. Pathobiology. 2017;84(5):258-63.

39. Nagai T, Kohsaka S, Okuda S, Anzai T, Asano K, Fukuda K. Incidence and prognostic significance of myocardial late gadolinium enhancement in patients with sarcoidosis without

cardiac manifestation. Chest. 2014;146(4):1064-72.

40. Kirkil G, Lower EE, Baughman RP. Predictors of Mortality in Pulmonary Sarcoidosis. Chest. 2018;153(1):105-13.

41. Boucly A, Cottin V, Nunes H, Jais X, Tazi A, Prevot G, et al. Management and long-term outcomes of sarcoidosis-associated pulmonary hypertension. Eur Respir J. 2017;50(4).

42. Jamilloux $Y$, Maucort-Boulch D, Kerever S, Gerfaud-Valentin M, Broussolle C, Eb M, et al. Sarcoidosis-related mortality in France: a multiple-cause-of-death analysis. Eur Respir J. 2016;48(6):1700-9. 
43. Handa T, Nagai S, Miki S, Fushimi Y, Ohta K, Mishima M, et al. Incidence of pulmonary hypertension and its clinical relevance in patients with sarcoidosis. Chest. 2006;129(5):1246-52.

44. Baughman RP, Engel PJ, Meyer CA, Barrett AB, Lower EE. Pulmonary hypertension in sarcoidosis. Sarcoidosis Vasc Diffuse Lung Dis. 2006;23(2):108-16.

45. Shorr AF, Helman DL, Davies DB, Nathan SD. Pulmonary hypertension in advanced sarcoidosis: epidemiology and clinical characteristics. Eur Respir J. 2005;25(5):783-8.

46. Galie N, Humbert M, Vachiery JL, Gibbs S, Lang I, Torbicki A, et al. 2015 ESC/ERS Guidelines for the diagnosis and treatment of pulmonary hypertension: The Joint Task Force for the Diagnosis and Treatment of Pulmonary Hypertension of the European Society of

Cardiology (ESC) and the European Respiratory Society (ERS): Endorsed by: Association for European Paediatric and Congenital Cardiology (AEPC), International Society for Heart and Lung Transplantation (ISHLT). Eur Respir J. 2015;46(4):903-75.

47. Janda S, Shahidi N, Gin K, Swiston J. Diagnostic accuracy of echocardiography for pulmonary hypertension: a systematic review and meta-analysis. Heart. 2011;97(8):612-22. 48. Duong H, Bonham CA. Sarcoidosis-associated Pulmonary Hypertension: Pathophysiology, Diagnosis, and Treatment. Clin Pulm Med. 2018;25(2):52-60. 49. Mitchell DN, Scadding JG, Heard BE, Hinson KF. Sarcoidosis: histopathological definition and clinical diagnosis. J Clin Pathol. 1977;30(5):395-408. 
50. Hawtin KE, Roddie ME, Mauri FA, Copley SJ. Pulmonary sarcoidosis: the 'Great Pretender'. Clin Radiol. 2010;65(8):642-50.

51. Walsh SL, Wells AU, Sverzellati N, Keir GJ, Calandriello L, Antoniou KM, et al. An integrated clinicoradiological staging system for pulmonary sarcoidosis: a case-cohort study. Lancet Respir Med. 2014;2(2):123-30.

52. Sharma S, Singh AD, Sharma SK, Tripathi M, Das CJ, Kumar R. Gallium-68 DOTA-NOC $\mathrm{PET} / \mathrm{CT}$ as an alternate predictor of disease activity in sarcoidosis. Nucl Med Commun. 2018;39(8):768-78.

53. Evidence-based indications for the use of PET-CT in the United Kingdom 2016. 2016. 54. Govender P, Berman JS. The Diagnosis of Sarcoidosis. Clinics in chest medicine.

2015;36(4):585-602.

55. Shorr AF, Torrington KG, Hnatiuk OW. Endobronchial biopsy for sarcoidosis: a prospective study. Chest. 2001;120(1):109-14.

56. Polychronopoulos VS, Prakash UB. Airway involvement in sarcoidosis. Chest. 2009;136(5):1371-80.

57. Lenique F, Brauner MW, Grenier P, Battesti JP, Loiseau A, Valeyre D. CT assessment of bronchi in sarcoidosis: endoscopic and pathologic correlations. Radiology. 1995;194(2):419-23.

58. Kantrow SP, Meyer KC, Kidd P, Raghu G. The CD4/CD8 ratio in BAL fluid is highly variable in sarcoidosis. The European respiratory journal. 1997;10(12):2716-21. 
59. Winterbauer RH, Lammert J, Selland M, Wu R, Corley D, Springmeyer SC. Bronchoalveolar lavage cell populations in the diagnosis of sarcoidosis. Chest. 1993;104(2):352-61.

60. Costabel U. CD4/CD8 ratios in bronchoalveolar lavage fluid: of value for diagnosing sarcoidosis? The European respiratory journal. 1997;10(12):2699-700.

61. Drent M, Mansour K, Linssen C. Bronchoalveolar lavage in sarcoidosis. Seminars in respiratory and critical care medicine. 2007;28(5):486-95.

62. Gilman MJ. Transbronchial biopsy in sarcoidosis. Chest. 1983;83(1):159.

63. Du Rand IA, Blaikley J, Booton R, Chaudhuri N, Gupta V, Khalid S, et al. British Thoracic Society guideline for diagnostic flexible bronchoscopy in adults: accredited by NICE.

Thorax. 2013;68 Suppl 1:i1-i44.

64. Ibrahim AS, Allangawi MH, Sattar HA, Mobyed HS, Almohammed AA. Indications, diagnostic yields and complications of transbronchial biopsy over 5 years in the State of Qatar. Saudi medical journal. 2005;26(4):641-5.

65. Milman N, Faurschou P, Munch EP, Grode G. Transbronchial lung biopsy through the fibre optic bronchoscope. Results and complications in 452 examinations. Respiratory medicine. 1994;88(10):749-53.

66. Anders GT, Johnson JE, Bush BA, Matthews JI. Transbronchial biopsy without fluoroscopy. A seven-year perspective. Chest. 1988;94(3):557-60. 
67. Puar HS, Young RC, Jr., Armstrong EM. Bronchial and transbronchial lung biopsy without fluoroscopy in sarcoidosis. Chest. 1985;87(3):303-6.

68. Wang KP, Nelson S, Scatarige J, Siegelman S. Transbronchial needle aspiration of a mediastinal mass: therapeutic implications. Thorax. 1983;38(7):556-7.

69. Hurter T, Hanrath P. Endobronchial sonography: feasibility and preliminary results. Thorax. 1992;47(7):565-7.

70. Adams K, Shah PL, Edmonds L, Lim E. Test performance of endobronchial ultrasound and transbronchial needle aspiration biopsy for mediastinal staging in patients with lung cancer: systematic review and meta-analysis. Thorax. 2009;64(9):757-62.

71. Holty JE, Kuschner WG, Gould MK. Accuracy of transbronchial needle aspiration for

mediastinal staging of non-small cell lung cancer: a meta-analysis. Thorax. 2005;60(11):949-55.

72. Agarwal R, Srinivasan A, Aggarwal AN, Gupta D. Efficacy and safety of convex probe EBUS-TBNA in sarcoidosis: a systematic review and meta-analysis. Respiratory medicine. 2012;106(6):883-92.

73. Dziedzic DA, Peryt A, Orlowski T. The role of EBUS-TBNA and standard bronchoscopic modalities in the diagnosis of sarcoidosis. The clinical respiratory journal. 2015.

74. Huang JA, Browning R, Wang KP. Counterpoint: Should Endobronchial Ultrasound Guide Every Transbronchial Needle Aspiration of Lymph Nodes? No. Chest.

2013;144(3):734-7. 
75. Wahidi MM, Yasufuku K. Point: Should Endobronchial Ultrasound Guide Every Transbronchial Needle Aspiration of Lymph Nodes? Yes. Chest. 2013;144(3):732-4.

76. von Bartheld MB, Dekkers OM, Szlubowski A, Eberhardt R, Herth FJ, in 't Veen JC, et al. Endosonography vs conventional bronchoscopy for the diagnosis of sarcoidosis: the GRANULOMA randomized clinical trial. Jama. 2013;309(23):2457-64.

77. Gupta D, Dadhwal DS, Agarwal R, Gupta N, Bal A, Aggarwal AN. Endobronchial ultrasound-guided transbronchial needle aspiration vs conventional transbronchial needle aspiration in the diagnosis of sarcoidosis. Chest. 2014;146(3):547-56.

78. Mehta AC, Almeida FA. Choose wisely: endobronchial ultrasound-guided transbronchial needle aspiration for sarcoidosis. Chest. 2014;146(3):530-2.

79.

Casoni GL, Tomassetti S, Cavazza A, Colby TV, Dubini A, Ryu JH, et al. Transbronchial lung cryobiopsy in the diagnosis of fibrotic interstitial lung diseases. Plos One.

2014;9(2):e86716.

80. Griff S, Schonfeld N, Ammenwerth W, Blum TG, Grah C, Bauer TT, et al. Diagnostic yield of transbronchial cryobiopsy in non-neoplastic lung disease: a retrospective case series. BMC pulmonary medicine. 2014;14:171.

81. Kropski JA, Pritchett JM, Mason WR, Sivarajan L, Gleaves LA, Johnson JE, et al. Bronchoscopic cryobiopsy for the diagnosis of diffuse parenchymal lung disease. Plos One. 2013;8(11):e78674. 
82. Rossides M, Kullberg S, Askling J, Eklund A, Grunewald J, Arkema EV. Sarcoidosis mortality in Sweden: a population-based cohort study. Eur Respir J. 2018;51(2).

83. Gribbin J, Hubbard RB, Le Jeune I, Smith CJ, West J, Tata LJ. Incidence and mortality of idiopathic pulmonary fibrosis and sarcoidosis in the UK. Thorax. 2006;61(11):980-5.

84. Ho JSY, Chilvers ER, Thillai M. Cardiac sarcoidosis - an expert review for the chest physician. Expert Rev Respir Med. 2019;13(6):507-20.

85. Ungprasert $\mathrm{P}$, Crowson CS, Matteson EL. Risk of cardiovascular disease among patients with sarcoidosis: a population-based retrospective cohort study, 1976-2013. Eur Respir J. 2017;49(2).

86. Khan NA, Donatelli CV, Tonelli AR, Wiesen J, Ribeiro Neto ML, Sahoo D, et al. Toxicity

risk from glucocorticoids in sarcoidosis patients. Respir Med. 2017;132:9-14.

87. Brito-Zeron P, Acar-Denizli N, Siso-Almirall A, Bosch X, Hernandez F, Vilanova S, et al. The Burden of Comorbidity and Complexity in Sarcoidosis: Impact of Associated Chronic Diseases. Lung. 2018;196(2):239-48.

88. Nowinski A, Puscinska E, Goljan A, Peradzynska J, Bednarek M, Korzybski D, et al. The influence of comorbidities on mortality in sarcoidosis: a observational prospective cohort study. Clin Respir J. 2017;11(5):648-56.

89. Iannuzzi MC, Rybicki BA, Teirstein AS. Sarcoidosis. N Engl J Med.

2007;357(21):2153-65. 
90. Uzunhan $\mathrm{Y}$, Nunes $\mathrm{H}$, Jeny F, Lacroix M, Brun S, Brillet PY, et al. Chronic pulmonary aspergillosis complicating sarcoidosis. Eur Respir J. 2017;49(6).

91. Atkins C, Wilson AM. Managing fatigue in sarcoidosis - A systematic review of the evidence. Chron Respir Dis. 2017;14(2):161-73.

92. Gibson GJ, Prescott RJ, Muers MF, Middleton WG, Mitchell DN, Connolly CK, et al. British Thoracic Society Sarcoidosis study: effects of long term corticosteroid treatment. Thorax. 1996;51(3):238-47.

93. Karakaya B, Kaiser Y, van Moorsel CHM, Grunewald J. Lofgren's Syndrome: Diagnosis, Management, and Disease Pathogenesis. Semin Respir Crit Care Med. 2017;38(4):463-76.

94. Thillai M, Chang W, Chaudhuri N, Forrest I, Ho LP, Lines S, et al. Sarcoidosis in the UK:

insights from British Thoracic Society registry data. BMJ Open Respir Res. 2019;6(1):e000357.

95. Thillai M, Potiphar L, Eberhardt C, Pareek M, Dhawan R, Kon OM, et al. Obstructive lung function in sarcoidosis may be missed, especially in older white patients. Eur Respir J. 2012;39(3):775-7.

96. Milman N, Graudal N, Grode G, Munch E. No effect of high-dose inhaled steroids in pulmonary sarcoidosis: a double-blind, placebo-controlled study. J Intern Med.

1994;236(3):285-90. 
97. Korsten P, Strohmayer K, Baughman RP, Sweiss NJ. Refractory pulmonary sarcoidosis - proposal of a definition and recommendations for the diagnostic and therapeutic approach. Clin Pulm Med. 2016;23(2):67-75.

98. Baughman RP, Nunes H, Sweiss NJ, Lower EE. Established and experimental medical therapy of pulmonary sarcoidosis. Eur Respir J. 2013;41(6):1424-38.

99. Paramothayan NS, Lasserson TJ, Jones PW. Corticosteroids for pulmonary sarcoidosis. Cochrane Database Syst Rev. 2005(2):CD001114.

100. Keenan CR, Salem S, Fietz ER, Gualano RC, Stewart AG. Glucocorticoid-resistant asthma and novel anti-inflammatory drugs. Drug Discov Today. 2012;17(17-18):1031-8.

101. Keir G, Wells AU. Assessing pulmonary disease and response to therapy: which test?

Semin Respir Crit Care Med. 2010;31(4):409-18.

102. Ledingham J, Gullick N, Irving K, Gorodkin R, Aris M, Burke J, et al. BSR and BHPR guideline for the prescription and monitoring of non-biologic disease-modifying anti-rheumatic drugs. Rheumatology (Oxford). 2017;56(6):865-8.

103. Vorselaars ADM, Wuyts WA, Vorselaars VMM, Zanen P, Deneer VHM, Veltkamp M, et al. Methotrexate vs azathioprine in second-line therapy of sarcoidosis. Chest. 2013;144(3):805-12.

104. Sahoo DH, Bandyopadhyay D, Xu M, Pearson K, Parambil JG, Lazar CA, et al. Effectiveness and safety of leflunomide for pulmonary and extrapulmonary sarcoidosis. Eur Respir J. 2011;38(5):1145-50. 
105. Hamzeh N, Voelker A, Forssen A, Gottschall EB, Rose C, Mroz P, et al. Efficacy of mycophenolate mofetil in sarcoidosis. Respir Med. 2014;108(11):1663-9.

106. Baughman RP, Lower EE. A clinical approach to the use of methotrexate for sarcoidosis. Thorax. 1999;54(8):742-6.

107. Baughman RP, Winget DB, Lower EE. Methotrexate is steroid sparing in acute sarcoidosis: results of a double blind, randomized trial. Sarcoidosis Vasc Diffuse Lung Dis. 2000;17(1):60-6.

108. Baughman RP, Costabel U, du Bois RM. Treatment of sarcoidosis. Clin Chest Med. 2008;29(3):533-48, ix-x.

109. Schutt AC, Bullington WM, Judson MA. Pharmacotherapy for pulmonary sarcoidosis: a

Delphi consensus study. Respir Med. 2010;104(5):717-23.

110. Cremers JP, Drent M, Bast A, Shigemitsu H, Baughman RP, Valeyre D, et al. Multinational evidence-based World Association of Sarcoidosis and Other Granulomatous Disorders recommendations for the use of methotrexate in sarcoidosis: integrating systematic literature research and expert opinion of sarcoidologists worldwide. Curr Opin Pulm Med. 2013;19(5):545-61.

111. Ridker PM, Everett BM, Pradhan A, MacFadyen JG, Solomon DH, Zaharris E, et al. Low-Dose Methotrexate for the Prevention of Atherosclerotic Events. N Engl J Med.

2019;380(8):752-62. 
112. Swigris JJ, Olson AL, Fischer A, Lynch DA, Cosgrove GP, Frankel SK, et al. Mycophenolate mofetil is safe, well tolerated, and preserves lung function in patients with connective tissue disease-related interstitial lung disease. Chest. 2006;130(1):30-6.

113. Coscia LA, Armenti DP, King RW, Sifontis NM, Constantinescu S, Moritz MJ. Update on the Teratogenicity of Maternal Mycophenolate Mofetil. J Pediatr Genet. 2015;4(2):42-55. 114. Baughman RP, Lower EE. Leflunomide for chronic sarcoidosis. Sarcoidosis Vasc Diffuse Lung Dis. 2004;21(1):43-8.

115. Baughman RP, Ohmichi M, Lower EE. Combination therapy for sarcoidosis.

Sarcoidosis Vasc Diffuse Lung Dis. 2001;18(2):133-7.

116. Xu Y, Lu JH, Ma L, Chen WJ, Lou X. Quantitative Measurement of Cerebral Blood Flow in

Enhanced Psedo-continuous Arterial Spin Labeling Perfusion Imaging in Patients with Intracranial Atherosclerotic Stenosis. Zhongguo Yi Xue Ke Xue Yuan Xue Bao.

2016;38(6):679-85.

117. Crouser ED, Lozanski G, Fox CC, Hauswirth DW, Raveendran R, Julian MW. The CD4+ lymphopenic sarcoidosis phenotype is highly responsive to anti-tumor necrosis factor-\{alpha\} therapy. Chest. 2010;137(6):1432-5.

118. Baughman RP, Drent $M$, Kavuru M, Judson MA, Costabel U, du Bois $R$, et al. Infliximab therapy in patients with chronic sarcoidosis and pulmonary involvement. Am J Respir Crit Care Med. 2006;174(7):795-802. 
119. Baughman RP, Lower EE, Drent M. Inhibitors of tumor necrosis factor (TNF) in sarcoidosis: who, what, and how to use them. Sarcoidosis Vasc Diffuse Lung Dis.

2008;25(2):76-89.

120. Skoie IM, Wildhagen K, Omdal R. Development of sarcoidosis following etanercept treatment: a report of three cases. Rheumatol Int. 2012;32(4):1049-53.

121. Nordgaard-Lassen I, Dahlerup JF, Belard E, Gerstoft J, Kjeldsen J, Kragballe K, et al. Guidelines for screening, prophylaxis and critical information prior to initiating anti-TNF-alpha treatment. Dan Med J. 2012;59(7):C4480.

122. Richeldi L, du Bois RM, Raghu G, Azuma A, Brown KK, Costabel U, et al. Efficacy and safety of nintedanib in idiopathic pulmonary fibrosis. N Engl J Med. 2014;370(22):2071-82.

123. King TE, Jr., Bradford WZ, Castro-Bernardini S, Fagan EA, Glaspole I, Glassberg MK, et al. A phase 3 trial of pirfenidone in patients with idiopathic pulmonary fibrosis. N Engl J Med. 2014;370(22):2083-92.

124. Flaherty KR, Wells AU, Cottin V, Devaraj A, Walsh SLF, Inoue Y, et al. Nintedanib in Progressive Fibrosing Interstitial Lung Diseases. N Engl J Med. 2019;381(18):1718-27. 125. George PM, Patterson CM, Reed AK, Thillai M. Lung transplantation for idiopathic pulmonary fibrosis. Lancet Respir Med. 2019;7(3):271-82.

126. Judson MA. Lung transplantation for pulmonary sarcoidosis. Eur Respir J. 1998;11(3):738-44. 
127. Taimeh Z, Hertz MI, Shumway S, Pritzker M. Lung transplantation for pulmonary sarcoidosis. Twenty-five years of experience in the USA. Thorax. 2016;71(4):378-9.

128. Kazerooni EA, Jackson C, Cascade PN. Sarcoidosis: recurrence of primary disease in transplanted lungs. Radiology. 1994;192(2):461-4.

129. Spruit MA, Thomeer MJ, Gosselink R, Troosters T, Kasran A, Debrock AJ, et al. Skeletal muscle weakness in patients with sarcoidosis and its relationship with exercise intolerance and reduced health status. Thorax. 2005;60(1):32-8.

130. Eklund A, du Bois RM. Approaches to the treatment of some of the troublesome manifestations of sarcoidosis. J Intern Med. 2014;275(4):335-49.

131. Birks J, Grimley Evans J. Ginkgo biloba for cognitive impairment and dementia.

Cochrane Database Syst Rev. 2009(1):CD003120.

132. Tan MS, Yu JT, Tan CC, Wang HF, Meng XF, Wang C, et al. Efficacy and adverse effects of ginkgo biloba for cognitive impairment and dementia: a systematic review and meta-analysis. J Alzheimers Dis. 2015;43(2):589-603.

133. Johnson SK, Diamond BJ, Rausch S, Kaufman M, Shiflett SC, Graves L. The effect of Ginkgo biloba on functional measures in multiple sclerosis: a pilot randomized controlled trial. Explore (NY). 2006;2(1):19-24.

134. Lower EE, Harman S, Baughman RP. Double-blind, randomized trial of dexmethylphenidate hydrochloride for the treatment of sarcoidosis-associated fatigue. Chest. 2008;133(5):1189-95. 
135. Lower EE, Malhotra A, Surdulescu V, Baughman RP. Armodafinil for sarcoidosis-associated fatigue: a double-blind, placebo-controlled, crossover trial. J Pain Symptom Manage. 2013;45(2):159-69.

136. Naz I, Ozalevli S, Ozkan S, Sahin H. Efficacy of a Structured Exercise Program for Improving Functional Capacity and Quality of Life in Patients With Stage 3 and 4 Sarcoidosis: A RANDOMIZED CONTROLLED TRIAL. J Cardiopulm Rehabil Prev. 2018;38(2):124-30.

137. Saketkoo LA, Karpinski A, Young J, Adell R, Walker M, Hennebury T, et al. Feasibility, utility and symptom impact of modified mindfulness training in sarcoidosis. ERJ Open Res. 2018;4(2).

138. Crawshaw AP, Wotton CJ, Yeates DG, Goldacre MJ, Ho LP. Evidence for association

between sarcoidosis and pulmonary embolism from 35-year record linkage study. Thorax. 2011;66(5):447-8.

139. Sharma OP, Johnson R. Airway obstruction in sarcoidosis. A study of 123 nonsmoking black American patients with sarcoidosis. Chest. 1988;94(2):343-6.

140. Harrison BD, Shaylor JM, Stokes TC, Wilkes AR. Airflow limitation in sarcoidosis--a study of pulmonary function in 107 patients with newly diagnosed disease. Respir Med. 1991;85(1):59-64.

141. Bradley B, Branley HM, Egan JJ, Greaves MS, Hansell DM, Harrison NK, et al. Interstitial lung disease guideline: the British Thoracic Society in collaboration with the 
Thoracic Society of Australia and New Zealand and the Irish Thoracic Society. Thorax. 2008;63 Suppl 5:v1-58.

142. Baughman RP, Judson MA. Relapses of sarcoidosis: what are they and can we predict who will get them? Eur Respir J. 2014;43(2):337-9.

143. Zappala CJ, Desai SR, Copley SJ, Spagnolo R, Cramer D, Sen D, et al. Optimal scoring of serial change on chest radiography in sarcoidosis. Sarcoidosis Vasc Diffuse Lung Dis. 2011;28(2):130-8.

144. Baumann MH, Strange C, Sahn SA. Do chest radiographic findings reflect the clinical course of patients with sarcoidosis during corticosteroid withdrawal? American Journal of Roentgenology. 1990;154(3):481-5.

145. Drent M, De Vries J, Lenters M, Lamers RJ, Rothkranz-Kos S, Wouters EF, et al. Sarcoidosis: assessment of disease severity using HRCT. Eur Radiol. 2003;13(11):2462-71. 146. Benamore R, Kendrick YR, Repapi E, Helm E, Cole SL, Taylor S, et al. CTAS: a CT score to quantify disease activity in pulmonary sarcoidosis. Thorax. 2016;71(12):1161-3.

147. Turner-Warwick M, McAllister W, Lawrence R, Britten A, Haslam PL. Corticosteroid treatment in pulmonary sarcoidosis: do serial lavage lymphocyte counts, serum angiotensin converting enzyme measurements, and gallium-67 scans help management? Thorax. 1986;41(12):903.

148. Gibson GJ. Sarcoidosis: old and new treatments. Thorax. 2001;56(5):336. 
149. Iliopoulou AA, A.; Murray, R. How to manage withdrawal of glucocorticoid therapy. Prescriber. 2013:23-9.

150. Dinsen S, Baslund B, Klose M, Rasmussen AK, Friis-Hansen L, Hilsted L, et al. Why glucocorticoid withdrawal may sometimes be as dangerous as the treatment itself. Eur J Intern Med. 2013;24(8):714-20.

151. Hopkins RL, Leinung MC. Exogenous Cushing's Syndrome and Glucocorticoid Withdrawal. Endocrinol Metab Clin North Am. 2005;34(2):371-84.

152. Valeyre D, Prasse A, Nunes H, Uzunhan Y, Brillet PY, Muller-Quernheim J. Sarcoidosis. Lancet. 2014;383(9923):1155-67. 